\title{
Capsaicin-loaded nanolipoidal carriers for topical application: design, characterization, and in vitro/ in vivo evaluation
}

This article was published in the following Dove Press journal:

International Journal of Nanomedicine

22 May 2017

Number of times this article has been viewed

\section{Xia-Rong Wang' \\ Si-Qian Gao' \\ Xiao-Qian Niu' \\ Long-Jian $\mathrm{Li}^{2}$ \\ Xiao-Ying Ying' \\ Zhong-Jie $\mathrm{Hu}^{2}$ \\ Jian-Qing $\mathrm{Gao}^{1,3}$}

'Institute of Pharmaceutics, College of Pharmaceutical Sciences, Zhejiang University, Hangzhou, ${ }^{2}$ Zhejiang Provincial Corps Hospital of Chinese People's Armed Police Forces, Jiaxing, Zhejiang, 3iangsu Engineering Research Center for New-Type External and Transdermal Preparations, Jiangsu, People's Republic of China
Correspondence: Jian-Qing Gao Institute of Pharmaceutics, College of Pharmaceutical Sciences, Zhejiang University, 866 Yuhangtang Road, Hangzhou 310058, Zhejiang, People's Republic of China

Tel/fax +8657188208436

Email gaojianqing@zju.edu.cn
Abstract: Capsaicin has been used in clinical applications for the treatment of pain disorders and inflammatory diseases. Given the strong pungency and high oil/water partition coefficient of capsaicin, capsaicin-loaded nanolipoidal carriers (NLCs) were designed to increase permeation and achieve the analgesic, anti-inflammatory effect with lower skin irritation. Capsaicin-loaded NLCs were prepared and later optimized by the Box-Behnken design. The physicochemical characterizations, morphology, and encapsulation of the capsaicin-loaded NLCs were subsequently confirmed. Capsaicin-loaded NLCs and capsaicin-loaded NLCs gel exhibited sustained release and no cytotoxicity properties. Also, they could significantly enhance the penetration amount, permeation flux, and skin retention amounts of capsaicin due to the application of NLCs. To study the topical permeation mechanism of capsaicin, 3,3'-dioctadecyloxacarbocyanine perchlorate (Dio) was used as a fluorescent dye. Dio-loaded NLCs and Dio-loaded NLCs gel could effectively deliver Dio up to a skin depth of 260 and $210 \mu \mathrm{m}$, respectively, primarily through the appendage route on the basis of version skin sections compared with Dio solution, which only delivered Dio up to $150 \mu \mathrm{m}$. In vivo therapeutic experiments demonstrated that capsaicin-loaded NLCs and capsaicin-loaded NLCs gel could improve the pain threshold in a dose-dependent manner and inhibit inflammation, primarily by reducing the prostaglandin E2 levels in the tissue compared with capsaicin cream and capsaicin solution. Meanwhile, skin irritation was reduced, indicating that application of NLCs could decrease the irritation caused by capsaicin. Overall, NLCs may be a potential carrier for topical delivery of capsaicin for useful pain and inflammation therapy.

Keywords: capsaicin-loaded NLCs, prescription optimization, analgesic, anti-inflammation, hot-plate test, carrageenan-induced paw edema

\section{Introduction}

Capsaicin is an alkaloid extracted from chili peppers that is widely used in clinical applications to treat pain and inflammatory diseases. ${ }^{1-5}$ Topical capsaicin acts in the skin to reduce pain and diminish cutaneous hypersensitivity by a process best described as the "defunctionalization" of nociceptor fibers. Defunctionalization can be achieved by repeated applications of capsaicin, which can render a loss of responsiveness in patients to a wide range of noxious stimuli. Due to its peculiar action on a subpopulation of sensory neurons, capsaicin has a strong and long-lasting anti-inflammatory analgesic effect which has been used as a therapy for the alleviation of various pain syndromes. ${ }^{1,6,7}$ Furthermore, capsaicin is used in other topical therapies for inflammatory diseases such as rheumatism. . $^{2,8}$

The time duration of capsaicin in the blood is rather short. The bioavailability of oral administration in humans has been reported through the following pharmacokinetic submit your manuscript Dovepress f
International Journal of Nanomedicine 2017:12 388|-3898

(c) (1) \& () 2017 Wang et al. This work is published and licensed by Dove Medical Press Limited. The full terms of this license are available at https://www.dovepress.com/terms.php (c) $\mathrm{BY}$ and incorporate the Creative Commons Attribution - Non Commercial (unported, v3.0) License (http://creativecommons.org/licenses/by-nc/3.0/). By accessing the work you hereby accept the Terms. Non-commercial uses of the work are permitted without any further permission from Dove Medical Press Limited, provided the work is properly attributed. For permission for commercial use of this work, please see paragraphs 4.2 and 5 of our Terms (https://www.dovepress.com/terms.php). 
parameters: a $C_{\max }$ of $2.5(0.1) \mathrm{ng} \mathrm{mL} \mathrm{mL}^{-1}$, a $T_{1 / 2}$ of $24.9(5.0)$ minutes, and a $T_{\max }$ of 47.1 (2.0) minutes. ${ }^{9}$ Interestingly, in vitro studies have shown that the metabolism of capsaicin in human skin is quite slow, ${ }^{10}$ and a longer elimination halflife was shown by a topically applied capsaicin patch which reflected its gradual release from the skin at the application site. Accordingly, topical application is an effective method for delivering therapeutic capsaicin for the treatment of different diseases given the special pharmacokinetic properties of capsaicin. ${ }^{11}$

Topical capsaicin formulations, such as low-concentration patches, lotions, and creams, have been accessible for daily skin application in most countries for many years. ${ }^{12}$ The combination of capsaicin formulations with nociceptors in the skin causes an initial excitation of the neurons and a period of enhanced sensitivity to noxious stimuli, which is usually reported as an itching, pricking, or burning sensation, indicating the appearance of thermal and mechanical hyperalgesia. ${ }^{13,14}$ These are the side effects that limit the clinical application of capsaicin. ${ }^{15}$ Furthermore, pure capsaicin is a lipophilic compound; indeed, it shows limited potential for topical delivery across skin. Thus, there is a need for more effective and safer vehicles for the application of capsaicin.

Nanolipoidal carriers (NLCs) are a promising type of topical drug carriers that can decrease dermal irritation ${ }^{16}$ and be appropriate for application on injured or inflamed skin because they are based on nonirritant and nontoxic lipids. ${ }^{17}$ Moreover, NLCs comprise solid and liquid lipids that lead to peculiar nanostructures with improved drug incorporation and release properties. ${ }^{18}$ It is demonstrated that liquid lipid can localize at the interface of the solid matrix and the surfactant layer or embed into the solid lipid matrix. These spatially different lipids do not form a highly ordered crystalline arrangement and thus provide more space for accommodating the encapsulated drugs. ${ }^{19,20}$ NLCs topically applied to the skin can also alter the drug penetration flux, provide drug deposition and localization, ${ }^{21}$ and even selectively permeate the stratum corneum (SC) by attaching themselves to the skin surface. In this way, adhesiveness and hydration are improved, and even lipid exchange between the lipid-based colloid carriers and the outermost layers of SC can be achieved. ${ }^{22}$ Rapid delivery of capsaicin can promote tolerability because some relative mechanisms of defunctionalization can occur very rapidly, whereas a loss of capsaicin responsiveness in vitro may develop within 20 seconds. ${ }^{23}$ Compared with polymeric nanoparticles, NLCs have an advantage in drug protection, sustained release, biodegradability, low toxicity, and the avoidance of organic solvents during preparation. More importantly, NLCs offer better prospects for industrial use with superiorities in terms of scale up, low cost, simple technology, etc. ${ }^{24}$ However, NLCs dispersion has low viscosity for topical application. To overcome this disadvantage, NLCs were incorporated into $\mathrm{Carbopol}^{\circledR} 934$ hydrogel in the present study to increase the convenience of usage and patient compliance.

For the acceptance of capsaicin in topical dosage form, it is necessary to circumvent its strong pungency. It was surmised that sustained release, improved permeation, and enhanced skin retention of capsaicin can improve the tolerability of capsaicin formulations. Consequently, the main objective of the present study was to produce a therapeutically effective formulation comprising synthetic lipids and capsaicin to decrease pain and inflammation with reduced skin irritation.

\section{Materials and methods Materials}

Capsaicin was kindly gifted by Ningbo Lihua Plant Extraction Technology Ltd (purity $>64 \%$ ) (Ningbo, People's Republic of China). Glyceryl monostearate was purchased from Aladdin, Inc. (Shanghai, People's Republic of China). Miglyol $^{\circledR} 812$ was obtained from Caelo (Hilden, Germany). Tween 80 (Shanghai Chemical Co., Ltd, Shanghai, People's Republic of China) was chosen as surfactant. Carbopol was provided by Hangzhou Keben Chemical Co., Ltd (Zhejiang, People's Republic of China). 3-(4,5-dimethyl-2-thiazolyl)2,5-diphenyl-2-H-tetrazolium bromide (MTT) was obtained from Aladdin, Inc. Annexin V-fluorescein isothiocyanate (FITC) Apoptosis Detection Kit was procured from KeyGEN BioTECH (Nanjing, People's Republic of China). 3,3'dioctadecyloxacarbocyanine perchlorate (Dio) was purchased from Beyotime Institute of Biotechnology (Jiangsu, People's Republic of China). Newborn calf serum was purchased from Gibco BRL (Gaithersburg, MD, USA). Rat PEG2 ELISA kit was purchased from Westang Biological Technology Co., Ltd (Shanghai, People's Republic of China). Double-distilled water was used throughout the studies. All other chemicals and solvents were of analytical reagent grade.

\section{Animals}

Female ICR mice (18-22 g), male Sprague Dawley (SD) rats (150-180 g), female SD rats (200 g), and Albino New Zealand rabbits $(2.5-3 \mathrm{~kg})$ were provided by Zhejiang University Experimental Animal Center, People's Republic of China. This study was approved by the Committee on Animal Use 
of Zhejiang University. The experimental procedures were in accordance with Zhejiang University guidelines for the welfare and ethics of the experimental animals. All the animals were grouped and housed in cages, provided free access to standard diet and water, and raised under constant conditions ( $12 \mathrm{~h}$ : $12 \mathrm{~h}$ light:dark cycle, $26^{\circ} \mathrm{C} \pm 1{ }^{\circ} \mathrm{C}$, and $50 \% \pm 15 \%$ relative humidity).

\section{Preparation of capsaicin-loaded NLCs}

Modified hot melt homogenization technique was used to prepare capsaicin-loaded NLCs by using a high shear homogenizer (Bandelin, Berlin, Germany) ${ }^{25}$ Briefly, lipids (consisting of solid lipid and liquid lipid materials) were melted with constant heating in an $80^{\circ} \mathrm{C}$ water bath. Then, capsaicin was added to the lipids and mixed completely by a magnetic stirrer which represented a clear lipid phase. Meanwhile, the aqueous phase containing Tween 80 was prepared and heated to the same temperature as the lipid phase. After dispersing the hot lipid phase into the hot aqueous phase drop by drop, the mixture was homogenized by the homogenizer at 8,000 rpm for 10 minutes. The obtained coarse hot oil-inwater emulsion was then ultrasonicated using a probe-type sonicator for 3 minutes. The resultant emulsion was cooled in an ice bath.

\section{Experimental design}

Response surface methodology using a 15-run, three-level, and three-factor Box-Behnken design was applied to optimize the experimental process, requiring minimal number of experiments to study the effects of independent variables on dependent variables (Design-Expert 8.0.6, Stat-Ease Inc., Minneapolis, MN, USA). The solid lipid and liquid lipid ratio $\left(\mathrm{X}_{1}\right)$, total amount of lipids $\left(\mathrm{X}_{2}\right)$, and amount of surfactant $\left(\mathrm{X}_{3}\right)$ were chosen as the independent variables, and they were assigned by level: $-1,0$, and +1 , namely, low, middle, and high values, respectively. Other parameters, that is, process conditions, sonication time, sonication amplitude, and final volume, were all set at fixed levels. The measured responses $\mathrm{Y}_{1}=$ entrapment efficiency $(\mathrm{EE} \%)$ and $\mathrm{Y}_{2}=$ particle size with restraints are described in Table S1. The study design comprised three center points and 12 factorial experiments to reduce the error. The Box-Behnken design was chosen in this experiment because of the fewer runs required versus the central composite design. ${ }^{26,27}$

Analysis of variance (ANOVA) was used to analyze the data in order to establish statistical validation such as the $R^{2}$ values and statistical significance of coefficients by the DesignExpert software. The analysis was considered significant when the $P$-values were $\leq 0.05$. Three-dimensional (3D) response surface plots which help explain the relationship between dependent variables and independent variables were further generated by the software. ${ }^{28}$

\section{Characterization of NLCs}

Particle size, polydispersity index (PDI), and zeta potential

Particle size, PDI, and zeta potential of the optimal capsaicinloaded NLCs were analyzed by Malvern Zetasizer Nano ZSP (Malvern Instruments Inc., Malvern, UK). Temperature and scattering angle were set at $25^{\circ} \mathrm{C} \pm 1^{\circ} \mathrm{C}$ and $90^{\circ}$, respectively. Sample was prepared by dispersing the capsaicin-loaded NLCs in double-distilled water before the determination. When the sample was used for zeta potential determination, the conductivity of the sample was adjusted by saline. The average size, PDI, and average zeta potential were attained from the software.

\section{Surface morphology}

The surface morphology of the optimal capsaicin-loaded NLCs was observed by transmission electron microscopy (TEM) (JEM-1200EX; JEOL, Tokyo, Japan). The required sample was prepared by covering the 400 -mesh copper grid with 40-fold diluted nanoparticle solution, then the copper grid was negative stained by $2 \%$ uranyl acetate solution.

\section{EE of capsaicin}

EE of the optimal capsaicin-loaded NLCs was determined using dialysis centrifugation method. ${ }^{29}$ Briefly, 1 mL NLCs dispersion was placed into a dialysis bag (molecular weight cutoff [MWCO] 8,000-14,000 Da) and two ends of the dialysis bag were tied. This dialysis bag was then put into the $10 \mathrm{~mL}$ centrifuge tube which was filled with $7 \mathrm{~mL}$ receptor phase (a mixture of ethanol and water at 1:1 ratio) and centrifuged for an hour at 6,000 rpm. The capsaicin content in the receptor phase was further estimated by high performance liquid chromatography (HPLC). The conditions of the HPLC method were as follows: mobile phase: acetonitrile and dilute phosphoric acid (1:1); injection volume $20 \mu \mathrm{L}$; detection wavelength $280 \mathrm{~nm}$; column: Diamonsil C18 (5 $\mu \mathrm{m}, 250 \times 4.6 \mathrm{~mm})$; column temperature $25^{\circ} \mathrm{C}$, and flow rate $1.0 \mathrm{~mL} / \mathrm{min}$. The total drug contained in the NLCs dispersion was determined by disrupting the mixture with methanol. EE\% was calculated using the following equation:

$$
\mathrm{EE} \%=\left(\mathrm{W}_{\text {total drug }}-\mathrm{W}_{\text {free drug }}\right) / \mathrm{W}_{\text {total drug }} \times 100 \%
$$


In the following experiments, all the capsaicin-loaded NLCs were used after purification by dialyzing with doubledistilled water for preset time.

\section{Fourier transform infrared spectroscopy (FTIR)}

Capsaicin (20 mg), unloaded NLCs (plain NLCs) freezedried powder, and capsaicin-loaded NLCs freeze-dried powder were weighed. FTIR determination were carried out by $\mathrm{KBr}$ tablet method using a PerkinElmer spectrum (version 10.03.08) spectrometer operated in the range of $500-4,000 \mathrm{~cm}^{-1}$ at a resolution of $4 \mathrm{~cm}^{-1}$.

\section{X-ray diffraction (XRD)}

The amorphous characteristic or crystalline state of capsaicin before and after formulation was studied by recording on a powder X-ray diffractometer (PANalytical $3 \mathrm{~kW}$ X'pert Powder; PANalytical, Nottingham, UK). Twenty milligrams of each sample was weighed and then loaded in polymethyl methacrylate stage. The samples were exposed to a $\mathrm{Cu}-\mathrm{K} \alpha$ radiation and scanned from $3 \theta$ to $50 \theta$ with $30 \mathrm{~mA}$ current.

\section{Preparation of capsaicin-loaded NLCs gel and capsaicin solution}

Capsaicin solution was prepared by dissolving a known amount of capsaicin in 10\% v/v ethanol in PEG400. In the preparation of capsaicin-loaded NLCs gel, an appropriate amount of carbopol was dispersed in capsaicin-loaded NLCs, stored overnight, and then neutralized with triethanolamine. The concentration of carbopol in the capsaicin-loaded NLCs gel was $1 \%$.

The preparation methods for Dio solution, Dio-loaded NLCs, and Dio-loaded NLCs gel which were used to investigate the percutaneous permeation of Dio by skin imaging have been described in the "Skin imaging studies" section.

\section{In vitro drug release study}

The in vitro drug release study of capsaicin cream, capsaicinloaded NLCs, capsaicin solution, and capsaicin-loaded NLCs gel was carried out by the dialysis bag method. ${ }^{30}$ One milliliter of NLCs dispersion and a weighed quantity of capsaicin-loaded NLCs gel with an equivalent amount of capsaicin were separately placed in the dialysis bag (MWCO 8,000-14,000 Da). The dialysis bags were tied at both ends and immersed individually into $50 \mathrm{~mL}$ centrifugation tubes which were filled with $30 \mathrm{~mL}$ phosphate-buffered saline (PBS, $\mathrm{pH}=7.4$ ) containing $0.5 \%$ Tween 80 . The centrifugation tubes were maintained in the incubator shaker at a constant temperature of $37^{\circ} \mathrm{C}$ for 48 hours. One milliliter of release medium was withdrawn intermittently at predetermined points in time. The same volume of a fresh and synthermal release medium was added to the system to maintain the sink condition. ${ }^{31}$ All the samples were further evaluated to quantify the content of capsaicin by HPLC.

\section{Cytotoxicity of capsaicin-loaded NLCs}

Cell and tissue exposure to nanoparticles is inevitable as nanoparticles are more widely applied. Consequently, the next step is to involve cell culture research to understand the cytotoxicity of capsaicin-loaded NLCs..$^{32}$ In the present study, the cell viability of capsaicin-loaded NLCs was evaluated by the MTT method and apoptosis experiment.

\section{MTT assay}

The cell viability of capsaicin-loaded NLCs was evaluated by MTT method. Human skin fibroblasts (HSF; CAS Type Culture Collection Committee Kunming Cell Bank, People's Republic of China) and human immortal keratinocyte line (HaCaT; Institute of Biochemistry and Cell Biology, Shanghai Institutes for Biological Sciences, Chinese Academy of Sciences, Shanghai, People's Republic of China) were individually seeded into 96-well plates at a density of $1 \times 10^{4}$ cells/well in $200 \mu \mathrm{L}$ Dulbecco's Modified Eagle's Medium (DMEM; full growth medium). The adherent cells were then treated with 5-120 $\mu \mathrm{M}$ capsaicin-loaded NLCs. After 24 hours, $40 \mu \mathrm{L} 5 \mathrm{mg} / \mathrm{mL}$ MTT solution was added to all wells and the cells were stained for 4 hours at $5 \% \mathrm{CO}_{2}$ and $37^{\circ} \mathrm{C}$. Then, the MTT encompassing growth medium was removed and $100 \mu \mathrm{L}$ DMSO was added to dissolve the formed formazan crystals for 10 minutes. The absorbance of the wells supernatant was measured spectrophotometrically in a microplate reader at $570 \mathrm{~nm}$ (BioTek Instruments, Inc., Winooski, VT, USA). Cells that were not treated with MTT solution were used as a blank group to calibrate the microplate reader and the untreated cells were used as control group which was equal to $100 \%$ cell viability. The relative cell viability was calculated by the formula:

$$
\text { Cell viability }(\%)=(\mathrm{abs})_{\text {sample }} /(\mathrm{abs})_{\text {control }} \times 100 \% \text {. }
$$

\section{Apoptosis experiment}

The cell apoptosis of capsaicin-loaded NLCs was evaluated by flow cytometry. HSF cells and $\mathrm{HaCaT}$ cells were seeded into 6-well plates at a density of $1 \times 10^{5}$ cells/well in $2 \mathrm{~mL}$ DMEM full growth medium. Allowing the adherence of cells overnight in the incubator, afterward, the growth medium was replaced by the medium which contained low, middle, and high concentrations of capsaicin-loaded 
NLCs. After 24 hours incubation, the medium was aspirated and the cells were washed twice with PBS (pH 7.4) and digested by EDTA-free trypsin. The cells suspension was stained with Annexin V-FITC/propidium iodide using an Annexin V-FITC Apoptosis Detection Kit (KeyGEN BioTECH) for flow cytometry by following the manufacturer's protocol.

\section{In vitro percutaneous permeation study Preparation of skin}

Female SD rats $(200 \mathrm{~g})$ were anesthetized. The hair in the dorsal surface was shaved by an electric razor without causing any damage to the skin. Appropriate amount of depilatory cream was applied over the entire backside for 5 minutes. Later, the depilatory cream was washed with warm water completely. The rats after depilation were raised under constant conditions for 24 hours to repair the damage caused by the depilatory cream. After sacrifice, the skin covering the dorsal surface was excised and the skin integrity test was performed on each skin sample to rule out any damage. Rat skins were stored at $-80^{\circ} \mathrm{C}$ for further studies and the storage time was not too long.

\section{In vitro penetration of capsaicin-based formulations} Prior to the experiment, the rat skins were thawed in PBS. The skin was microscopically examined to ensure the integrity of the surface. Then, the skin was mounted onto a Franz diffusion cell (Shanghai Kai Science and Technology Trade Co., Ltd, People's Republic of China). The area of the rat skin subjected to different capsaicin preparations in the donor chamber was $2.83 \mathrm{~cm}^{2}$, and the volume of the receiver fluid was $6.8 \mathrm{~mL}$. Tested formulations equaling $1 \mathrm{mg}$ capsaicin were applied evenly on the skin surface. The receiver compartment was filled with receptor fluid (ethanol and PBS $1: 1)$, maintained at $37^{\circ} \mathrm{C} \pm 0.5^{\circ} \mathrm{C}$ and stirred at $300 \mathrm{rpm}$. The bubbles in the receiver compartment had to be removed completely. The skin was covered with a Teflon film. At predetermined times, $1 \mathrm{~mL}$ of receptor fluid was withdrawn, and the same volume of fresh fluid was added into the receiver compartment. The amount of capsaicin penetrating through the skin at different times was quantified by HPLC. The cumulative penetration amount $(\mathrm{Q})\left(\mu \mathrm{g} / \mathrm{cm}^{2}\right)$ was calculated using the following formula:

$$
\mathrm{Q}=\mathrm{V}\left(\mathrm{C}_{1}+\mathrm{C}_{2}+\ldots \mathrm{C}_{\mathrm{i}}\right) / \mathrm{A}
$$

$\mathrm{C}_{1}, \mathrm{C}_{2}$, and $\mathrm{C}_{\mathrm{i}}$ represent the capsaicin concentrations in the first, second, and last points, respectively. A and $\mathrm{V}$ represent the area and volume of the Franz diffusion cell (in $\mathrm{cm}^{2}$ and $\mathrm{mL}$ ), respectively. The permeability coefficient $(J)\left(\mu \mathrm{g} \mathrm{cm}^{-2} \mathrm{~h}^{-1}\right)$ is the linear regression of $\mathrm{Q}$ after the release reaches steady. The slope intersect with the $\mathrm{x}$-axis is the $\mathrm{T}_{\text {lag }}$. After 24 hours, the entire treated skin surface was collected with a biopsy punch and used for further studies.

\section{Skin imaging study}

Skin imaging study was performed according to previous work with modifications. ${ }^{33,34}$ In brief, confocal laser scanning microscopy (CLSM) was used to study the skin distribution and permeation mechanism of the Dio-NLCs, Dio-NLCs gel, and Dio solution. The amount of Dio dye applied remained the same for all preparations. The experimental process was performed in a similar way as described earlier. After 24 hours permeation, the skin sections of different formulations were visualized with CLSM (BX61W1-FV1000; Olympus Inc., Tokyo, Japan) using water and a $10 \times$ objective throughout the study period. After confocal laser scanning, the skins were embedded with optimal cutting temperature, and vertical, full-thickness skin sections were collected using a cryotome (Leica CM1950; Leica Microsystems, Wetzlar, Germany). The sections were visualized with a fluorescence microscope using a 10× objective (Nikon ECLIPSE Ti, Tokyo, Japan).

\section{Skin retention of capsaicin}

To detect the concentration of drug detained in the different rat skin layers, the SC, epidermal (Epi), and dermis were separated individually using the modified method. ${ }^{25,35,36} \mathrm{SC}$ was separated by adhesive tape (Scotch ${ }^{\mathrm{TM}}$; 3M Co., Two Harbors, MN, USA) using the tape-stripping method. Tape stripping was performed on the skin surface until the tape was clean. All the tapes were collected and then placed in a beaker. The capsaicin contained in the SC was extracted from the tapes using methanol.

The Epi sheet was separated from the left skin with a surgical scalpel after heating in a $60^{\circ} \mathrm{C}$ water bath for 1.5 minutes; then, the single Epi and dermis were both cut into small pieces and transferred into a centrifuge tube. Three hundred microliters of PBS ( $\mathrm{pH}=7.4)$ was added to all the processed skin layers and they were heated in a boiling water bath for 10 minutes. After boiling, all the samples were cooled down to room temperature, and $700 \mu \mathrm{L}$ of acetonitrile was added. The tubes were sonicated for 1 hour by a water sonicator and then vortexed for 1 minute. At the final point, all the samples were centrifuged at 15,000 rpm for 15 minutes. The supernatant was analyzed for the determination of the capsaicin content using HPLC. 


\section{In vivo study}

Hot-plate test

The in vivo analgesic study was conducted by employing the hot-plate test. ${ }^{37}$ Female ICR mice ( $\left.20 \mathrm{~g}\right)$ were divided into eight groups $(n=8)$ randomly. All the mice were habituated to equipment and conditions for 2 minutes once per day in a constant environment prior to determining the baseline nociceptive threshold. In the hot-plate test, the metal hot plate was maintained at $52^{\circ} \mathrm{C} \pm 0.5^{\circ} \mathrm{C}$, and the response time was recorded, including paw licking, paw flutter, or jumping. Seven different capsaicin formulations, explicitly, capsaicin cream $(0.75 \mathrm{mg} / \mathrm{mL})$, capsaicin-loaded NLCs $(1.5,0.75$, and $0.375 \mathrm{mg} / \mathrm{mL})$, and capsaicin-loaded NLCs gel $(1.5,0.75$, and $0.375 \mathrm{mg} / \mathrm{mL}$ ), and the control group were applied every day to the paws of the rats and the nociceptive threshold was measured after 14 days of administration. A maximum response time of 30 seconds was set to avoid any damage to the mice. After each measurement, the metal plate was cleaned with sanitary paper to remove traces of urine and feces.

\section{Carrageenan-induced paw edema in the rats}

The anti-inflammatory activity of the capsaicin-based preparations was evaluated using carrageenan-induced hind paw edema method, which was developed using a previously reported method. ${ }^{38,39} \mathrm{In}$ brief, acute inflammatory paw edema of male SD rats (150-180 g) was produced by subcutaneous injection of $0.2 \mathrm{~mL} 1 \%(\mathrm{w} / \mathrm{w})$ homogeneous suspension of carrageenan solution per rat in the left hind paw. The SD rats were divided randomly into six groups $(n=10)$ : votalin cream, capsaicin-loaded NLCs, capsaicin-loaded NLCs gel, capsaicin cream, capsaicin solution, and the blank control. The amounts of capsaicin in the different capsaicin-based formulations were equivalent to $0.75 \mathrm{mg} / \mathrm{mL}$. Each sample $(0.2 \mathrm{~mL})$ per rat was topically administered to the hind paw where the carrageenan solution had been injected. The changes in paw volume were directly measured using a plethysmometer comprising two vertical, water-filled, and interconnecting perspex tubes at 1, 2, 4, 6, 8, 10, 24 hours postinjection of carrageenan.

\section{Determination of prostaglandin E2 (PGE2) levels in the paw tissue}

The inflamed hind paws were collected at the end of the experiment, and the tissues from the carrageenan-injected paws were peeled. Subsequently, the obtained tissue was weighed, chopped, and placed in tubes. PBS was added to the tissue to obtain a concentration equivalent to $5 \mathrm{~mL} / \mathrm{g}$ of paw tissue and homogenized. Then, the tissue homogenate was sonicated for 30 seconds. Finally, the homogenates were centrifuged for 30 minutes at $12,000 \mathrm{rpm}$ and stored at $4{ }^{\circ} \mathrm{C}$. The rat ELISA kit was used to determinate the PGE2 levels in the supernatants.

\section{Skin irritation test}

The capsaicin-based formulations were assessed for skin irritation using the Draize method of scoring. ${ }^{40}$ Considering worldwide efforts to reduce animal testing, a minimum number of Albino New Zealand rabbits were used in this test. Hair was shaved off, and the surface of the dorsal skin was cleared with hair removal cream to expose sufficient test areas $\sim 3 \times 3 \mathrm{~cm}^{2}$ in size which could accommodate three test sites on the dorsal skin at 24 hours prior to the study. One site was used for studying the irritation on damaged skin using a sterile blade to partially break SC. The second site was used for studying the irritation on normal skin, and the third was used as the control. The rabbits were randomly divided into four groups: $1.5 \mathrm{mg} / \mathrm{mL}$ capsaicin solution, $0.75 \mathrm{mg} / \mathrm{mL}$ capsaicin solution, $1.5 \mathrm{mg} / \mathrm{mL}$ capsaicin-loaded NLCs, and $1.5 \mathrm{mg} / \mathrm{mL}$ capsaicin-loaded NLCs gel. The skin reactions were observed 1 hour before and after each administration and 24, 48, and 72 hours after the end of the administration.

\section{Statistical analysis}

All data are represented as the means and standard deviation (mean \pm standard deviation). Statistical significance in terms of multiple group comparisons was verified by a one-way ANOVA using SPSS software (version 22; IBM Corporation, Armonk, NY, USA). A direct comparison between two groups was conducted by the two-tailed and unpaired Student's $t$-test in Microsoft Excel. Differences were considered very significant at $P<0.01$ and significant at $P<0.05$.

\section{Results and discussion Optimization of the prescription of capsaicin-loaded NLCs by Design-Expert}

The response surface methodology used a 15-run, threefactor, three-level Box-Behnken design to optimize the process. The chosen independent variables mentioned above considerably affected the observed responses. The experimental results are shown in Table S2. Polynomial equations with the main effects and interaction factors were generated by Design-Expert software. The polynomial equation fitted to $\mathrm{EE}$ is given by the following quadratic equation:

$$
\begin{aligned}
\mathrm{Y}_{1}= & 90.5+0.97 \mathrm{X}_{1}+0.53 \mathrm{X}_{2}+2.38 \mathrm{X}_{3}-2.5 \mathrm{X}_{1}^{2} \\
& -1.75 \mathrm{X}_{2}^{2}-2.5 \mathrm{X}_{3}^{2}
\end{aligned}
$$


Table I Summary of results of regression analysis of responses

\begin{tabular}{|c|c|c|c|c|}
\hline Response & F-value & $\begin{array}{l}\text { Lack of } \\
\text { fit F-value }\end{array}$ & $R^{2}$ & $\begin{array}{l}\text { Adequate } \\
\text { precision }^{\mathrm{a}}\end{array}$ \\
\hline EE (\%) & 7.74 & 0.3249 & 0.8531 & 8.130 \\
\hline PS (nm) & 10.03 & 0.0415 & 0.9093 & 10.994 \\
\hline
\end{tabular}

Notes: a Measures signal to noise ratio. Ratio $\geq 4$ is desirable. Abbreviations: $\mathrm{EE}$, entrapment efficiency; PS, particle size.

The polynomial equation fitted to the particle size is given by the following quadratic equation:

$$
\begin{aligned}
Y_{2}= & 125.54+2 X_{1}+15 X_{2}-15.75 X_{3}+13.5 X_{1} X_{2} \\
& -11 X_{1} X_{3}+23.81 X_{1}^{2}+12.31 X_{3}^{2}
\end{aligned}
$$

The results of regression analysis of responses are shown in Table 1. The parameters indicated that quadratic Equation 1 was significant and could be used to predict the optimal prescription. However, the significance of the fit of quadratic Equation 2 was insufficient, indicating that Equation 2 needs further validation. The 3D response surface analysis is shown in Figure 1.

\section{Optimization and validation}

The optimal formulation of capsaicin-loaded NLCs was conducted by establishing the criteria of minimum particle size and maximum entrapment efficacy. Three groups of capsaicin-loaded NLCs with the surmised levels of formulation factors were classified to endorse the validity of the optimization prescription. The compositions of optimized formulation were $450 \mathrm{mg}$ solid lipid, $450 \mathrm{mg}$ liquid lipid, and $300 \mathrm{mg}$ Tween 80 . EE and particle size of the optimized formulation were $90.5 \% \pm 0.9 \%$ and $115 \pm 5 \mathrm{~nm}$, respectively, which denoted good agreement with the predicted values. The predicted values of EE $\%$ and particle size for the optimal formulation were $90.72 \%$ and $114 \mathrm{~nm}$, respectively, and the $\%$ bias of the optimized formulation acquired was $0.24 \%$ for $\mathrm{EE} \%$ and $-0.88 \%$ for particle size. The quadratic equations $\mathrm{Y}_{1}$ and $\mathrm{Y}_{2}$ could be used to optimize the prescription.

\section{Physicochemical characterizations of capsaicin-loaded NLCs}

A morphology study of capsaicin-loaded NLCs was conducted by TEM. The images revealed that the capsaicinloaded NLCs were nearly spherical $\sim 100 \mathrm{~nm}$ (Figure 2A). The size distribution of the capsaicin-loaded NLCs was uniform (Figure 2B). The entrapment efficacy, zeta potential, and average particle size of optimum capsaicin-loaded NLCs were $91.6 \% \pm 1.3 \%,-17.0 \pm 0.4 \mathrm{mV}$, and $119 \pm 4 \mathrm{~nm}$ with a PDI of $0.181 \pm 0.09$, respectively (Figure 2C). The PDI indicated the minimum tendency to aggregate and the homogeneity of the size distribution when it was $<0.2 .^{41,42}$ However, the size distribution of the capsaicin-loaded NLCs was irregular, which was slightly different from the previous result obtained by TEM. This phenomenon may be due to the state of determination; TEM showed the morphological size of nanoparticles in the solid state, whereas the laser particle size analyzer measured the hydrodynamic diameter of nanoparticles in the liquid state. ${ }^{43,44}$

To confirm the entrapment of capsaicin in NLCs, FTIR and XRD studies were conducted. FTIR spectroscopy was applied to characterize the molecular dynamics of the components. Characteristic peaks of capsaicin were found to exist at $3,331,2,969,2,924,2,853$, and $1,640 \mathrm{~cm}^{-1}$ as revealed in the FTIR spectra (Figure 2D). These peaks were found to be slightly shifted to $3,436,2,930,2,849,1,735$, and $1,464 \mathrm{~cm}^{-1}$ in the FTIR spectra of capsaicin-loaded NLCs. This result confirmed the entrapment of capsaicin in NLCs.

An XRD study was conducted to investigate the crystalline or amorphous characteristic of the capsaicin-loaded NLCs. The XRD profile of pure capsaicin showed characteristic crystalline peaks at $2 \theta$ positions of $5.861^{\circ}, 8.662^{\circ}$, $11.74^{\circ}, 16.421^{\circ}, 19.842^{\circ}, 23.281^{\circ}$, and $26.498^{\circ}$. The XRD spectra of plain NLCs only showed characteristic peaks of a lipid mixture at $2 \theta$ positions of $5.501^{\circ}, 19.159^{\circ}, 19.558^{\circ}$, $20.518^{\circ}, 21.42^{\circ}, 22.979^{\circ}$, and $23.461^{\circ}$. Meanwhile, the XRD spectra of capsaicin-loaded NLCs showed characteristic peaks of a lipid mixture as well. The shift and decline in the intensity of the characteristic peaks of capsaicin in the XRD profiles of capsaicin-loaded NLCs suggested that capsaicin was successfully entrapped in NLCs, in accordance with the FTIR results (Figure 2E).

\section{In vitro drug release study}

In vitro release profiles of the capsaicin-based preparations are demonstrated in Figure 2F. Capsaicin-loaded NLCs showed a sustained release of $>48$ hours. This could be attributed to both the dissolution and diffusion of capsaicin as well as the gradual degradation of the carrier matrix in the release medium. ${ }^{45}$ The capsaicin-loaded NLCs gel also showed sustained release, but carbopol slightly affected the release profile of capsaicin-loaded NLCs, and the cumulative release of capsaicin shifted from $82 \%$ to $59.2 \%$. The sustained release of capsaicin-loaded NLCs gel could be attributed to the network of carbopol, which influenced the release rate of the dissolution of capsaicin liquid medium by delaying free diffusion. The release profile of capsaicin cream 
A i

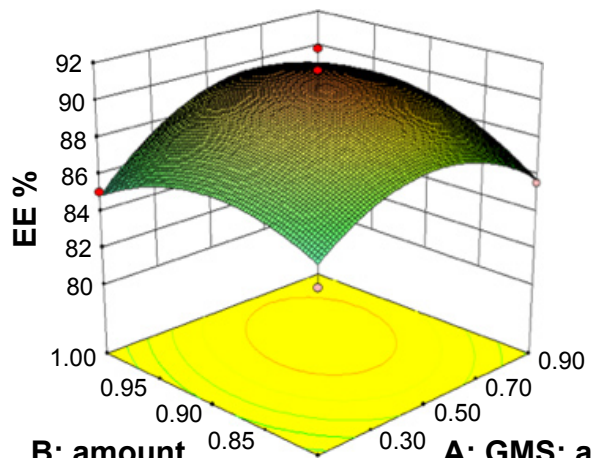

B: amount of lipid $(g)$ ii

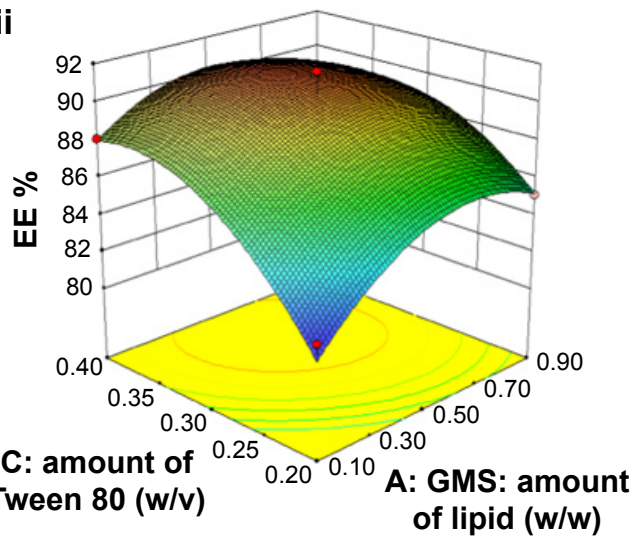

iii

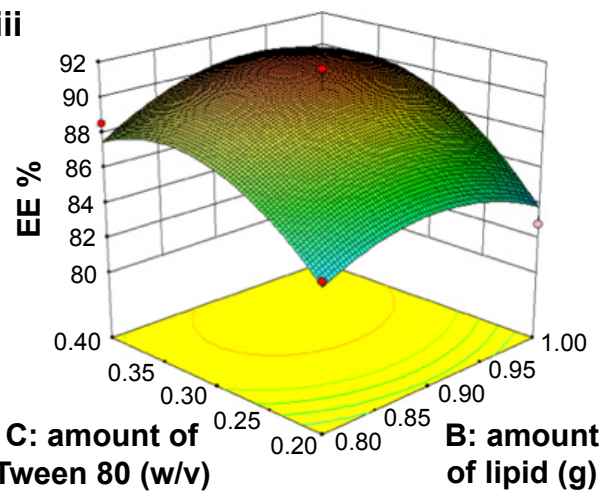

- Design points above predicted value o Design points below predicted value 91.6

81.9
B i

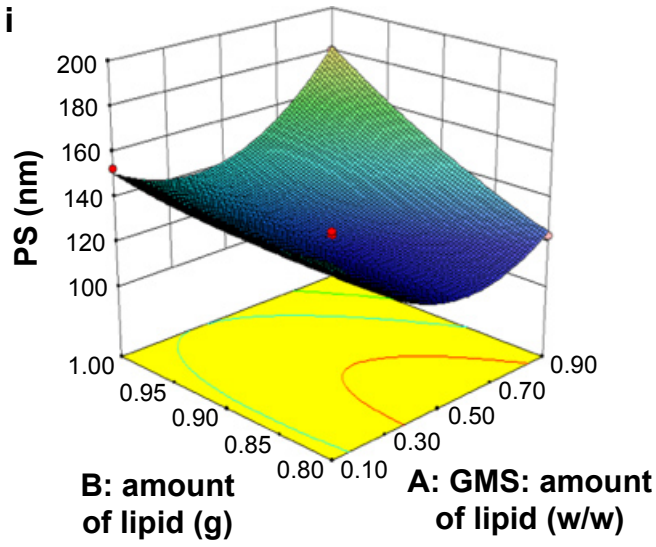

iii

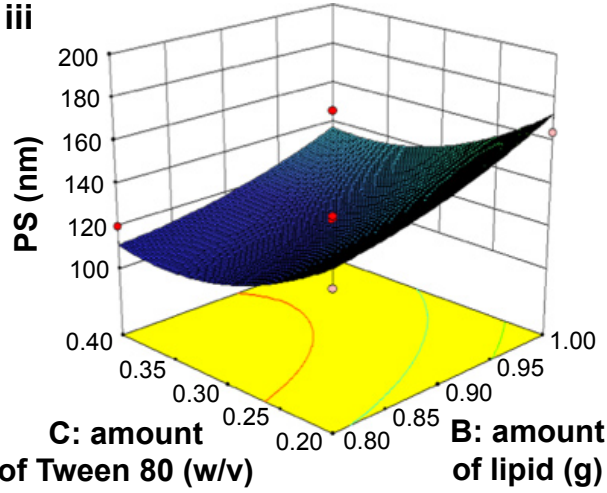

ii

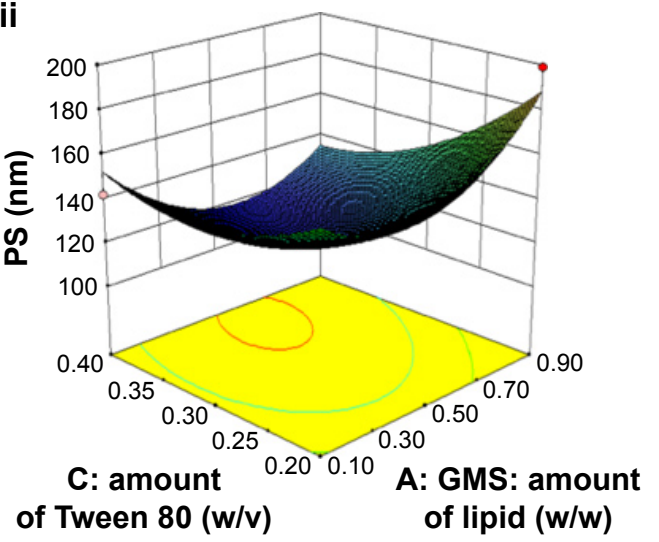

- Design points above predicted value Q Design points below predicted value 199

120

Figure I 3D response surface analysis. (A) 3D response surface plot for effect of GMS: amount of lipids, amount of lipids and amount of Tween 80 on entrapment efficiency. (B) 3D response surface plot for effect of GMS: amount of lipids, amount of lipids and amount of Tween 80 on particle size. Abbreviations: EE, entrapment efficiency; PS, particle size; 3D, three dimensional. 
A

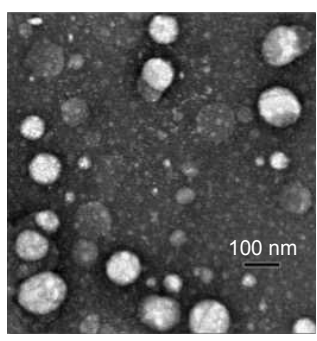

B

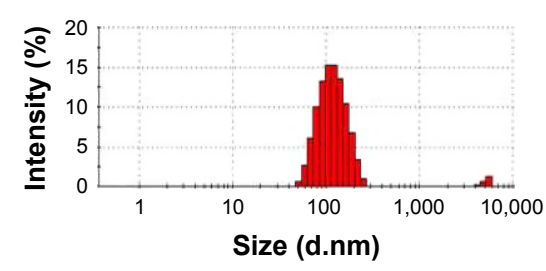

C

\begin{tabular}{ll}
\hline Capsaicin-loaded NLCs & Parameters \\
\hline Size & $121 \pm 4 \mathrm{~nm}$ \\
PDI & $0.181 \pm 0.09$ \\
Zeta potential & $-17 \pm 0.4 \mathrm{mV}$ \\
Encapsulation & $91.6 \% \pm 1.3 \%$ \\
\hline
\end{tabular}

F 120

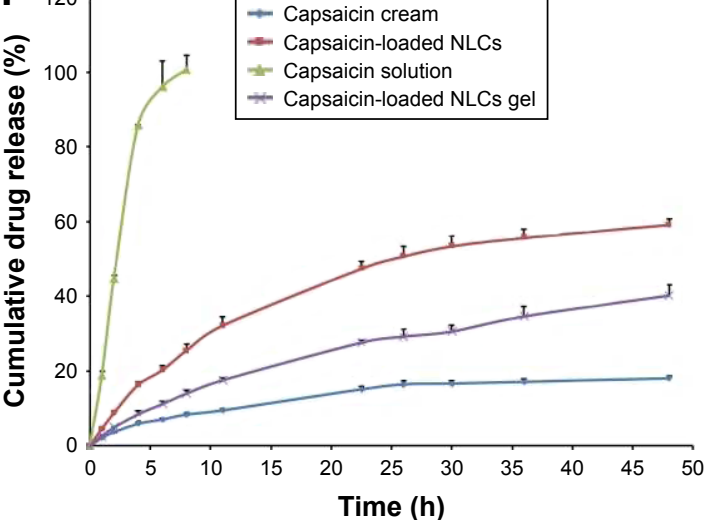

D

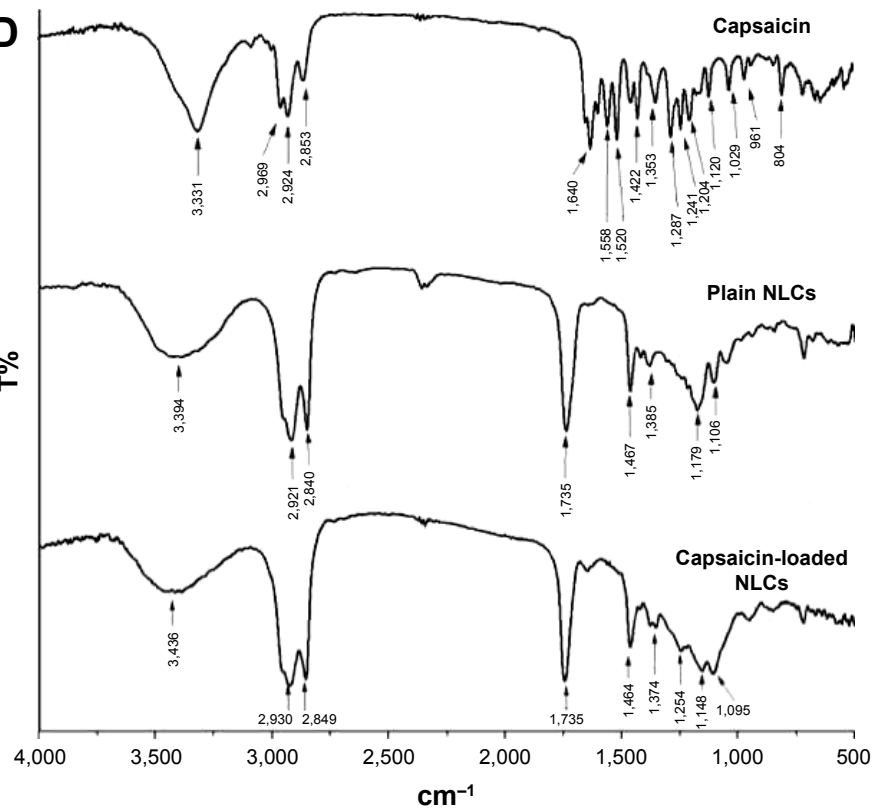

E
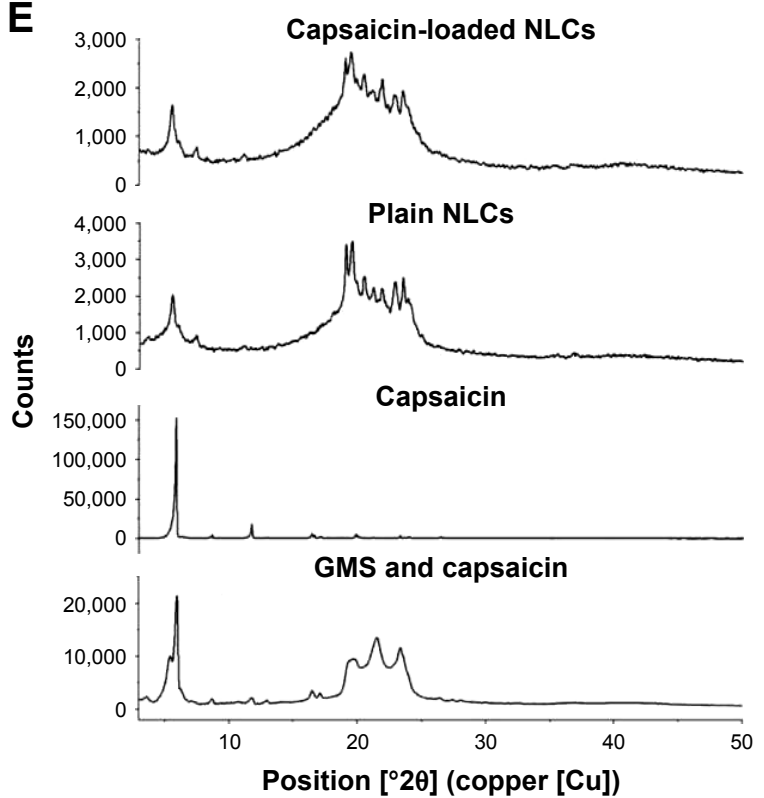

Figure 2 (A) Capsaicin-loaded-NLCs observed by TEM. (B) Size distribution of the capsaicin-loaded-NLCs. (C) Characterization of the capsaicin-loaded-NLCs. (D) FTIR profile for capsaicin-based formulations. (E) XRD profile for capsaicin-based formulations. (F) In vitro drug release profile of capsaicin-based formulations. The release medium used in vitro drug release study is $30 \mathrm{~mL}$ PBS $(\mathrm{pH}=7.4)$ contained $0.5 \%$ Tween 80 . Data presented here is the mean \pm SD $(n=3)$.

Abbreviations: NLCs, nanolipoidal carriers; PDI, polydispersity index; TEM, transmission electron microscopy; FTIR, Fourier transform infrared spectroscopy; XRD, X-ray diffraction; PBS, phosphate buffer saline; SD, standard deviation; GMS, Glycerol monostearate; T\%, transmittance\%.

showed a sustained release, as well. However, the cumulative drug release was only half of that of the capsaicin-loaded NLCs gel. The capsaicin solution showed fast release, and the percentage of drug cumulative release almost reached $100 \%$ by 8 hours.

\section{Cytotoxicity of capsaicin-loaded NLCs}

To evaluate the cytotoxicity of capsaicin-loaded NLCs, MTT assay and apoptosis experiment were performed after 24 hours of treatment. The outcomes showed that cell viability of the HaCaT and HSF cells was $>80 \%$ when treated with up to $120 \mu \mathrm{M}$, indicating that capsaicin-loaded NLCs did not cause cytotoxicity (Figure 3A), and the HaCaT and HSF cells after 24 hours incubation with 120, 60, and $10 \mu \mathrm{M}$ capsaicin-loaded NLCs did not show any cytotoxicity as well (Figure 3B and C). With the above cytotoxicity profile of capsaicin-loaded NLCs, it can be anticipated that capsaicin-loaded NLCs are safe to use.

\section{In vitro percutaneous permeation study} In vitro permeation of capsaicin formulations

The in vitro permeation profile performed at the end of 24 hours is shown in Figure 4A. The initial permeations of capsaicin-loaded NLCs, capsaicin-loaded NLCs gel, and capsaicin cream occurred at a similar percutaneous speed. However, capsaicin solution was below the detection limits of HPLC detection in the first 10 hours which may be attributed to its high oil/water partition coefficient. After 24 hours permeation, the percent drug dose permeation of capsaicin-loaded NLCs, capsaicin-loaded NLCs gel, and 

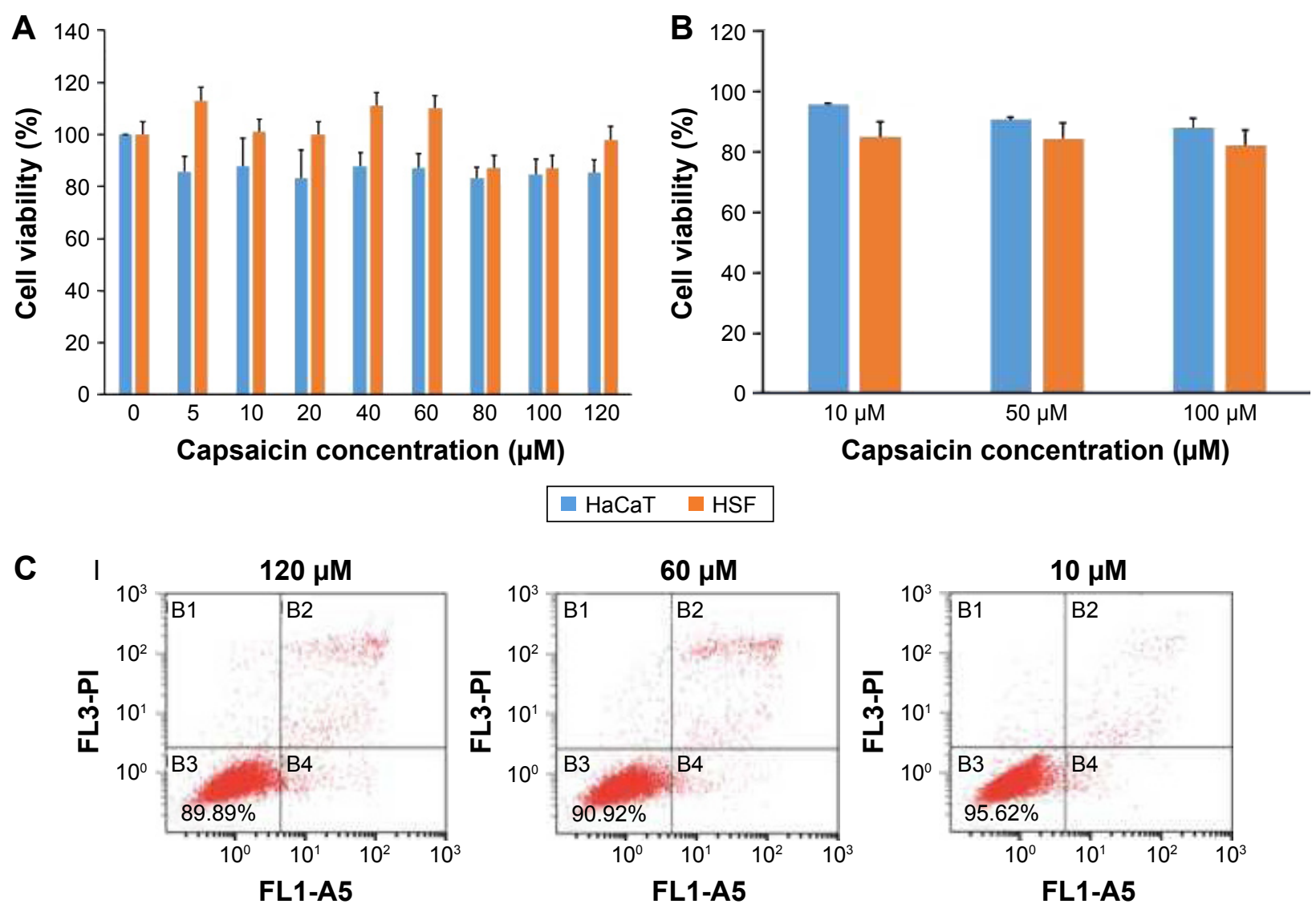

HaCaT $\mathrm{HSF}$

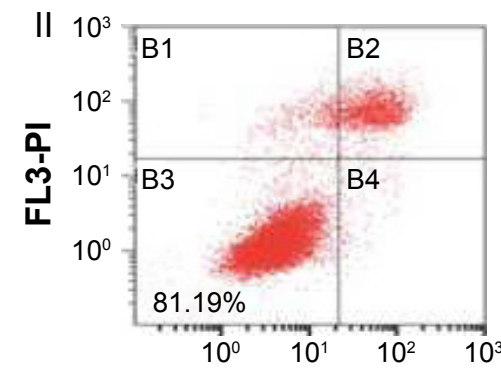

FL1-A5
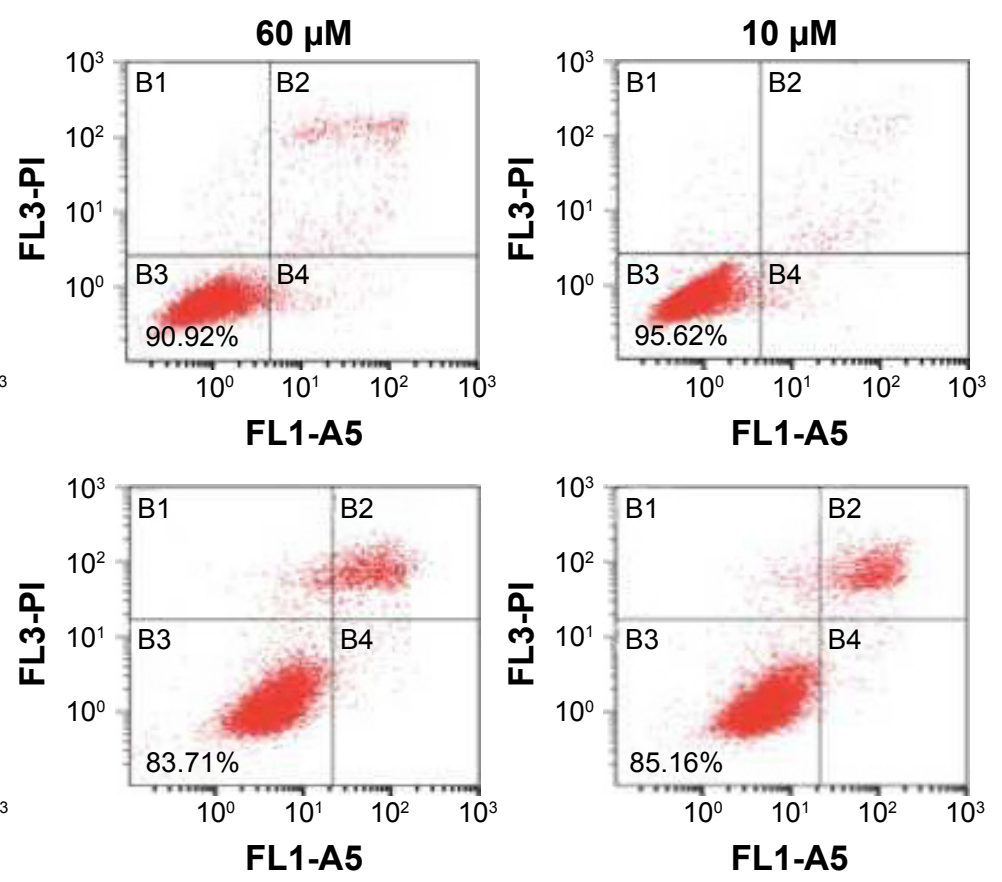

Figure 3 Cytotoxicity of capsaicin-loaded-NLCs. (A) Relative cell viability of HaCaT cells and HSF cells on incubation with different concentrations of capsaicin-loaded-NLCs after 24 h. Data represent mean \pm SD $(n=6)$. (B) Quantitative analysis of relative cell viability of HaCaT cells and HSF cells using flow cytometry. Data represent mean \pm SD $(\mathrm{n}=3)$. (C) Apoptosis experiments of capsaicin-loaded-NLCs in HaCaT cells and HSF cells. (I) Relative cell viability rate of HaCaT cells. (II) Relative cell viability rate of HSF cells. In MTT assay, all the cells were incubated with different concentrations of capsaicin-loaded-NLCs for $24 \mathrm{~h}$. Data represent mean \pm SD ( $\mathrm{n}=6$ ). In Apoptosis experiments, all the cells were incubated with different concentrations of capsaicin-loaded-NLCs for 24 h. Data represent mean \pm SD ( $n=3$ ).

Abbreviations: HSF, human skin fibroblast; PI, propidium iodide; NLCs, nanolipoidal carriers; SD, standard deviation; HaCaT, human immortal keratinocyte line; FL, fluorescence.

capsaicin cream was $8.4 \%, 7.2 \%$, and $5.1 \%$, respectively, which were significantly $>0.6 \%$ for capsaicin solution. Furthermore, the percent drug dose permeation across the skin of capsaicin-loaded NLCs was significantly higher than the other formulations. The capsaicin cream only showed ordinary permeation unlike a patch on capsaicinloaded NLCs gel and capsaicin-loaded NLCs. It was speculated that the presence of chemical permeation enhancers in the capsaicin cream was the reason for its better permeation profile than the capsaicin solution. Permeation coefficients of capsaicin released from capsaicin-based formulations are shown in Table 2. The cumulative drug permeated (Q) were $29.7 \mu \mathrm{g} \cdot \mathrm{cm}^{-2}$ and $25.6 \mu \mathrm{g} \cdot \mathrm{cm}^{-2}$ for capsaicin-loaded-NLCs and capsaicin-loaded-NLCs-Gel which were significantly higher than the $17.9 \mu \mathrm{g} \cdot \mathrm{cm}^{-2}$ and $2.2 \mu \mathrm{g} \cdot \mathrm{cm}^{-2}$ for capsaicinCream and capsaicin-Solution. The permeation flux rate $(J)$ of capsaicin-loaded NLCs and capsaicin-loaded NLCs gel was 14.4- and 12.2-fold faster than capsaicin solution. According to the above results, NLCs could enhance the cumulative permeation and permeation flux rate of capsaicin; 

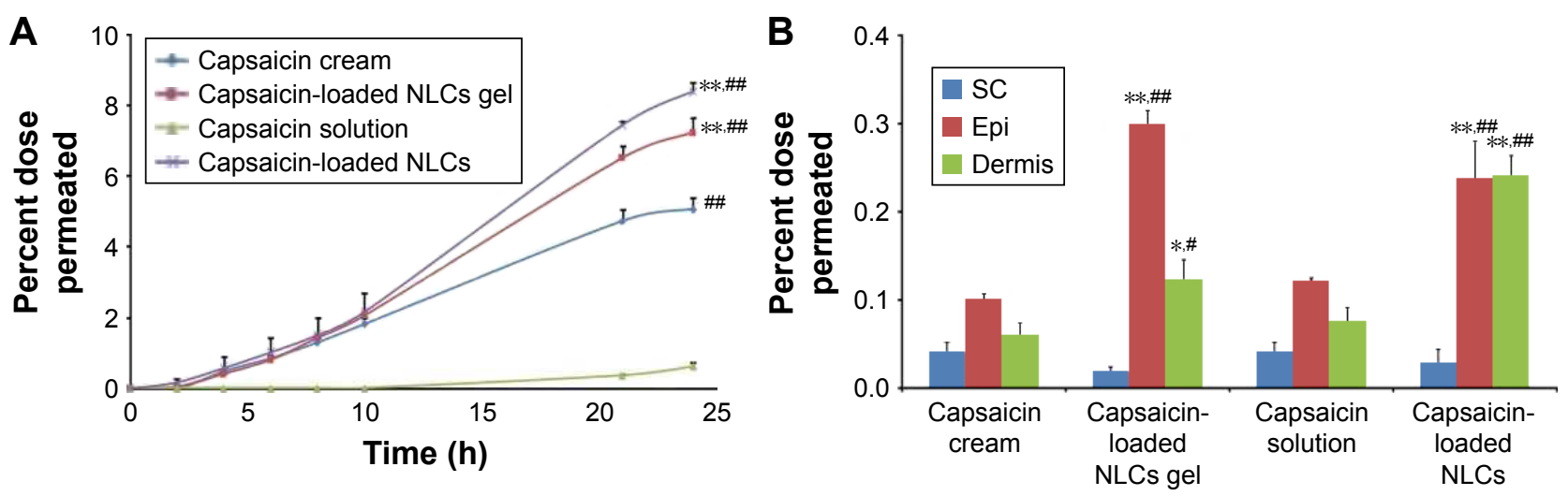

Figure 4 (A) In vitro drug permeation profile of capsaicin-based formulations. (B) In vitro skin retention profiles of capsaicin-based formulations. The In vitro drug permeation study and the skin retention study were performed using Franz diffusion cells with Sprague Dawley rat dorsal skin for $24 \mathrm{~h}$. The data represent \% dose permeated from capsaicin-based formulations. Data presented here is the mean $\pm S D(n=6)$; *significant capsaicin-based formulations against capsaicin-Cream, ** $P<0.0$; ; ${ }^{*}$ ignificant capsaicin-based formulations against capsaicin-Solution, \#P<0.01.

Abbreviations: NLCs, nanolipoidal carriers; SD, standard deviation; SC, stratum corneum; Epi, epidermis.

thus, it was surmised that it may be due to the nano-size, composition, and sustained release of NLCs. The nano-size of NLCs allowed the particles to be in close contact with the outer layer skin cells to increase the fluidity of the skin and then compelled the nanoparticles into the deeper skin layer. ${ }^{46}$ The components composing the NLCs were reported to have a permeation ability which could facilitate the penetration of nanoparticles into the deeper skin layer. ${ }^{47}$ The sustained release of NLCs also contributed to the enhancement of the permeation of capsaicin. ${ }^{34}$

\section{Skin retention of capsaicin}

To detect the concentration of capsaicin in different skin layers, drug extraction from SD dorsal skin was further studied using HPLC. The result showed that NLCs had an influence on the skin permeation of capsaicin after 24 hours of incubation. A sizeable amount of capsaicin was deposited into both the Epi and dermis from the NLCs-based formulations. The percentage of capsaicin deposited in the SC, Epi, and dermis from capsaicin-loaded NLCs was $0.029 \pm 0.014,0.237 \pm 0.043$, and $0.241 \% \pm 0.022 \%$, respectively. Interestingly, the amount of permeated capsaicin from the capsaicin-loaded NLCs deposited in the dermis was significantly higher than for other groups. This may increase the therapeutic effect of capsaicin because the skin nerve fibers mainly exist in the dermis. The percentage of capsaicin deposited in the SC, Epi, and dermis from the capsaicin-loaded NLCs gel was $0.019 \pm 0.005,0.299 \pm 0.016$, and $0.122 \% \pm 0.023 \%$, respectively, which was slightly lower than for the capsaicin-loaded NLCs solution. However, the capsaicin deposited in the dermis was still significantly higher than the capsaicin cream and capsaicin solution. Capsaicin-loaded NLCs could deliver 1.96- and 3.2-fold more capsaicin than the capsaicin solution and 2.35- and 4.02-fold more capsaicin than the capsaicin cream in Epi and dermis. Identically, the capsaicin-loaded NLCs gel could deliver 2.47- and 1.63-fold more capsaicin than the capsaicin solution and 2.96- and 2.03-fold more capsaicin than the capsaicin cream in Epi and dermis (Figure 4B). The enhanced skin retention of capsaicin from NLCs-based formulations may be due to the occlusive effect of NLCs on the skin surface. It increased the penetration of capsaicin by close contact with SC because NLCs possess unique characteristics such as high specific surface area and thus adhesive properties. ${ }^{48}$

\section{Skin imaging studies}

The incorporation of Dio fluorescence dye in NLCs was imaged under confocal microscopy by scanning the z-axis. The permeation efficacy of Dio-loaded NLCs is presented

Table 2 Percutaneous permeation coefficient of capsaicin release from the capsaicin-based formulations

\begin{tabular}{lllll}
\hline Formulations & $\mathbf{Q}\left(\mu \mathbf{g ~ c m}^{-2}\right)$, mean $\pm \mathbf{S D}$ & $\mathbf{J}\left(\mu \mathbf{g ~ c m}^{-2} \mathbf{h}^{-1}\right)$ & $\mathbf{T}_{\text {lag }}(\mathbf{h})$ & Enhancement ratio \\
\hline Capsaicin-loaded NLCs & $29.7 \pm 0.8$ & 18.7 & 0.59 & 14.4 \\
Capsaicin-loaded NLCs gel & $25.6 \pm 1.4$ & 15.8 & 0.54 & 12.2 \\
Capsaicin cream & $17.9 \pm 1.1$ & 10.3 & 0.37 & 7.9 \\
Capsaicin solution & $2.2 \pm 0.3$ & 1.3 & $\mathrm{I}$ & $\mathrm{I}$ \\
\hline
\end{tabular}

Abbreviations: NLCs, nanolipoidal carriers; $Q$, cumulative penetration amount; J, permeability coefficient; $T_{\text {lag }}$, time lag. 


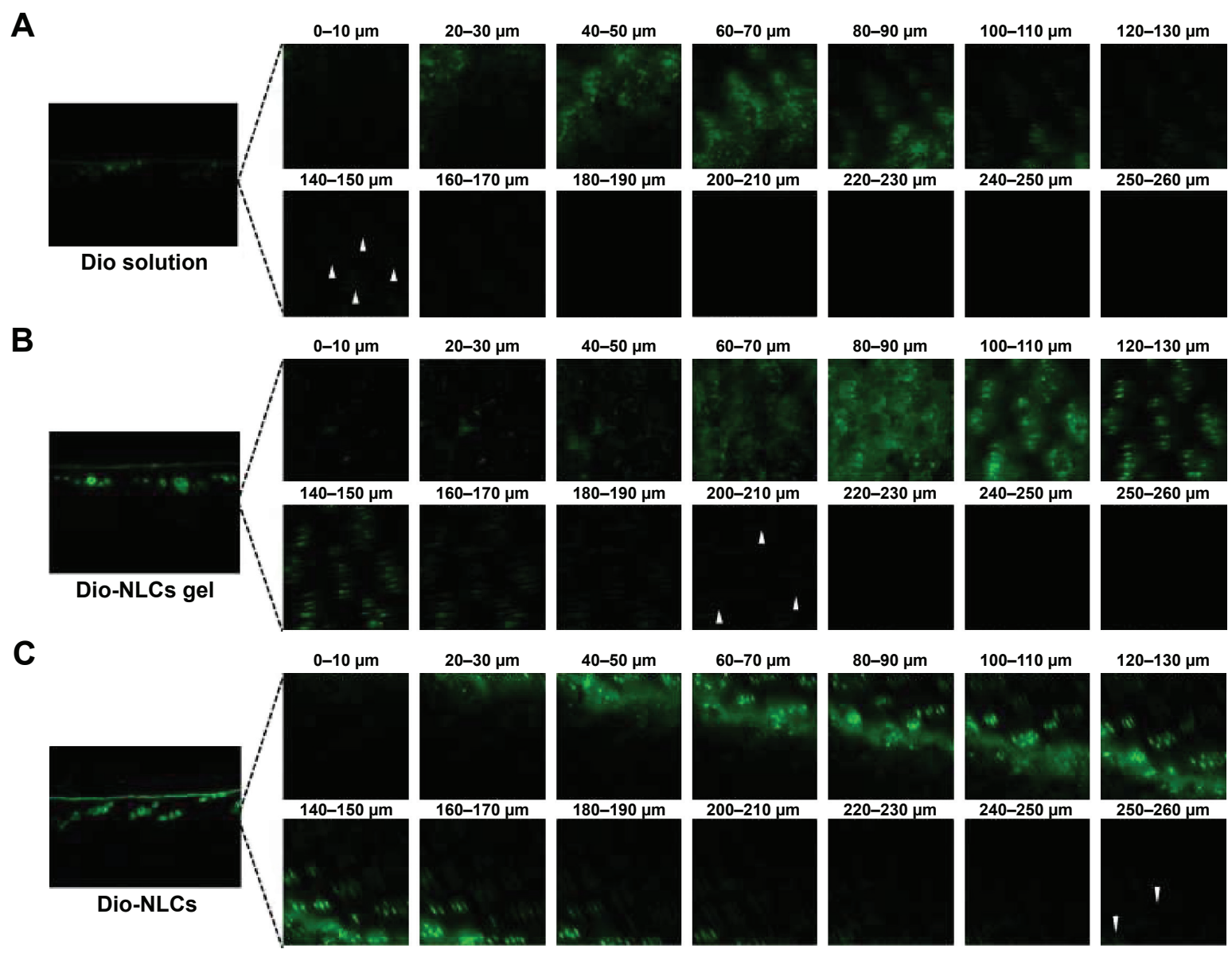

Figure 5 (A-C) CLSM images of Dio-based formulations. The skin imaging study was performed using Franz diffusion cells with Sprague Dawley rat dorsal skin for 24 h. The surplus drug was wiped off by cotton and the skin was directly observed under CLSM. Furtherly, vertical skin sections of Dio-based different formulations were observed for skin associated fluorescence.

Notes: (A) White triangles indicate the Dio solution can penetrate up to a skin depth of I50 $\mu \mathrm{m}$. (B) White triangles indicate the Dio-NLCs gel can deliver Dio up to 2 I $0 \mu \mathrm{m}$. (C) White triangles indicate the Dio-NLCs solution can deliver Dio up to $260 \mu \mathrm{m}$.

Abbreviations: Dio, 3,3'-dioctadecyloxacarbocyanine perchlorate; NLCs, nanolipoidal carriers; SD, standard deviation; CLSM, confocal laser scanning microscope.

in Figure 5. The images evidently demonstrate that the incorporation of Dio in NLCs can improve the skin permeation of Dio. The images show that all the fluorescence from the different Dio-based formulations decreased with the increase in skin depth. The fluorescence intensity of Dio solution was observed up to $20 \mu \mathrm{m}$, it declined at 120 $\mu \mathrm{m}$, and then diminished at $150 \mu \mathrm{m}$. Meanwhile, higher intense fluorescence signals were observed deep in the dermal region with a depth of 210 and $260 \mu \mathrm{m}$ from DioNLCs gel and Dio-NLCs, respectively. Vertical sections for different Dio-based formulations are separately displayed in Figure 5. The fluorescence intensity of Dio from Dio-NLCs gel and Dio-NLCs was much higher than the Dio solution and the small green fluorescent dots were the inside region of the appendages route. By superimposing the fluorescence microscopy image and the microscopy image, it was surmised that the NLCs promoted the permeation of Dio mainly through the appendages route. In conclusion, NLCs could significantly promote the penetration of drugs into deeper skin through the appendage route as can NLCs combined with the hydrogel.

\section{In vivo study}

\section{Therapeutic effect}

The experimental results of the hot-plate test are shown in Figure 6A. The pain threshold of the $1.5 \mathrm{mg} / \mathrm{mL}$ capsaicinloaded NLCs group, $0.75 \mathrm{mg} / \mathrm{mL}$ capsaicin-loaded NLCs group, and $1.5 \mathrm{mg} / \mathrm{mL}$ capsaicin-loaded NLCs gel group increased significantly after 14 days of continuous administration compared with the basic threshold. From the above results, it was inferred that the analgesic effect of capsaicin was dose dependent. Furthermore, the capsaicin-based NLCs formulations showed better analgesic efficacy than capsaicin cream at the same concentration. It is presumed that this result was mainly due to the better permeation ability and higher skin retention of the capsaicin-based NLCs preparations. The capsaicin-loaded NLCs showed more potency than the capsaicin-loaded NLCs gel, which may also be 

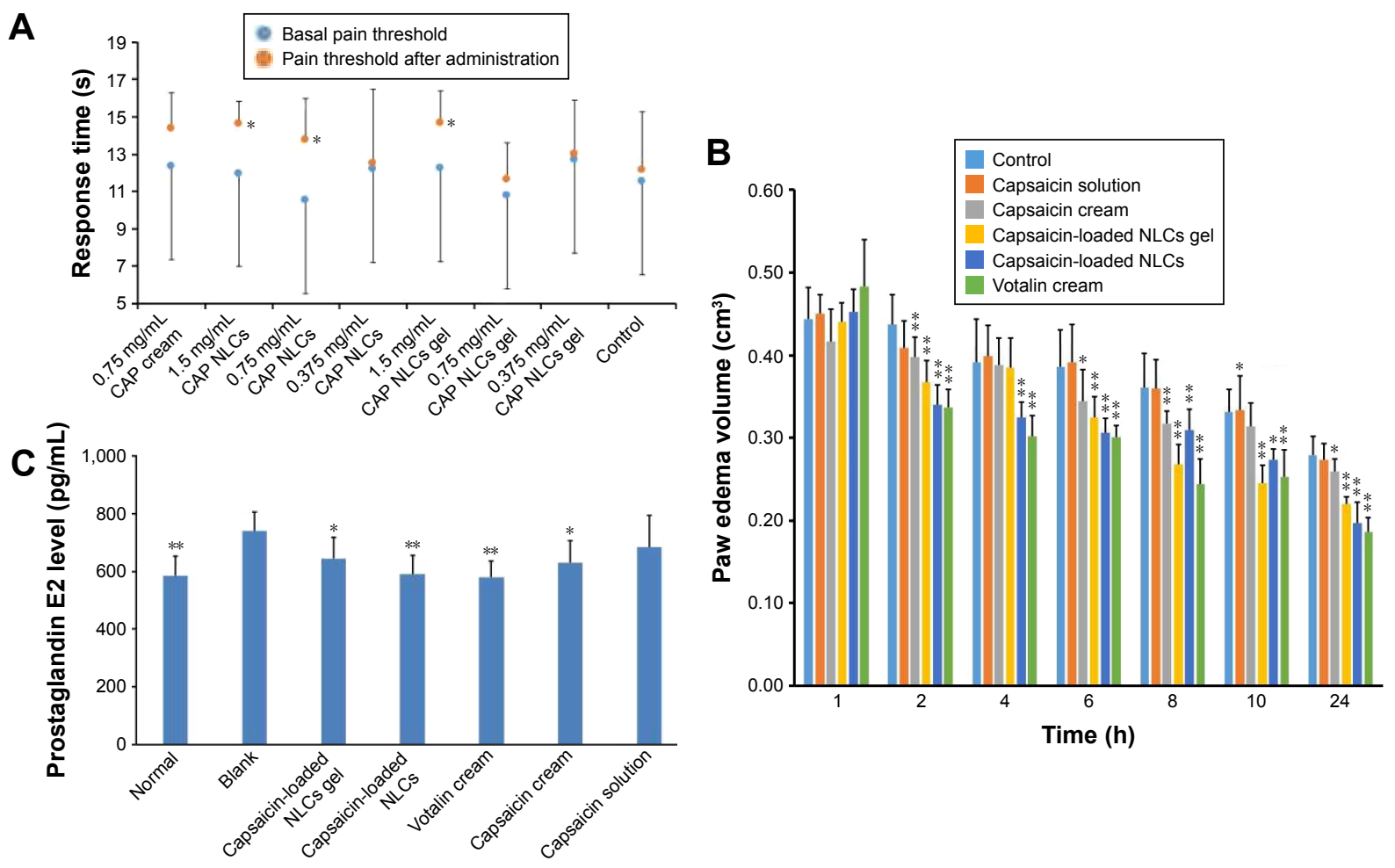

Figure 6 (A) The analgesic effect of capsaicin on the pain which is induced by hot-plate. (B) The volume of the inflammatory action induced hind paw edema in rats after different treatments at different times. (C) The PGE2 levels in the homogenates of the inflammatory injury paws of rats. The hot-plate test were conducted after 14 days of administration. Data presented here is the mean $\pm S D(n=8)$. *significant pain threshold after administration against basal pain threshold, $P<0.05$. In anti-inflammation study, data presented here is the mean $\pm S D(n=10)$; *significant other formulations against control, ${ }^{*} P<0.05, * * P<0.01$. In PGE2 level determination, it was determined after $24 \mathrm{~h}$ treatment by different formulations. Data presented here is the mean $\pm S D(n=10)$; *significant other formulations against the Blank group, $* P<0.05 ; * * P<0.01$.

Abbreviations: NLCs, nanolipoidal carriers; CAP, capsaicin; SD, standard deviation; PGE2, prostaglandin E2.

due to the faster permeation speed and greater retention of capsaicin in the skin. Further, carrageenan-induced paw edema was used in rats to study the anti-inflammatory effect of capsaicin. Previous work had proven that an injection of carrageenan could successfully induce acute inflammation. Figure 6B shows the volume of the left hind paw at 1, 2, 4, $6,8,10$, and 24 hours post-application of capsaicin-based formulations and votalin cream. The results suggested the efficacy of capsaicin-based formulations and votalin cream in paw edema inhibition. Except for the blank control and capsaicin solution, the inhibition effect in edema was observed in other treatments groups. Without doubt, votalin cream showed the best anti-inflammatory effect. However, the capsaicin-loaded NLCs group showed the best paw edema inhibition among the capsaicin-based formulations, followed by capsaicin-loaded NLCs gel and capsaicin cream. The results demonstrated that with NLCs as the delivery carrier, anti-inflammation of capsaicin was significantly enhanced due to the developed permeation ability. Incorporating the NLCs into hydrogel slightly decreased the inflammatory inhibition effect of capsaicin which may be mainly due to the slower permeation speed and less retention of capsaicin in the skin.

\section{Determination of PGE2 levels in the paw tissue}

The development of carrageenan-induced edema is biphasic. The first phase occurs within 1 hour which is attributed to the release of neurotransmitter molecules. The second phase (over $1 \mathrm{~h}$ ) is mediated by an increased release in PGEs in the inflammatory tissue. ${ }^{49}$ This study determined the PGE2 level to investigate the mechanism. Injection of carrageenan into the left hind paw of rats induced a significant increase of PGE2 in the paw compared with PGE2 in normal tissue. After treatment, the PGE2 levels in the other three capsaicinbased formulations were significantly reduced, except for the PGE2 level in the capsaicin solution group (Figure 6C). The results suggested that the edema inhibition effect of capsaicin worked possibly through the reduction of the PGE2 level, and the capsaicin-loaded NLCs displayed stronger efficiency in inhibiting the PGE2 level than the capsaicin-loaded NLCs gel and capsaicin cream which may be due to the faster permeation speed and greater capsaicin retention.

\section{Skin irritation test}

The results obtained from the normal and injured skin irritation test revealed that the $0.75 \mathrm{mg} / \mathrm{mL}$ capsaicin solution showed slight skin irritation on normal as well as injured 


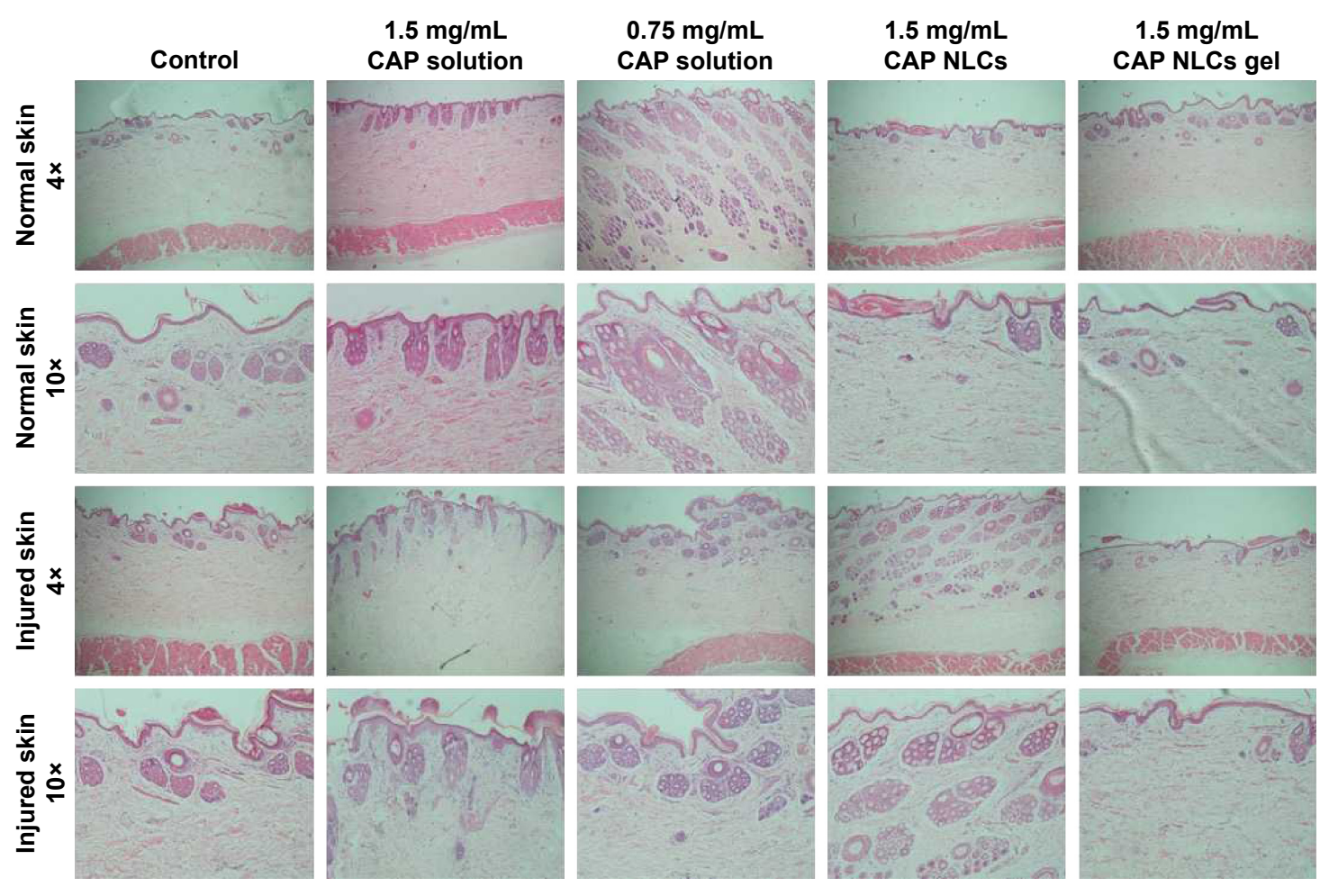

Figure $7 \mathrm{HE}$ staining of the dorsal skin of rabbits treated with different capsaicin-based formulations and different concentrations. Abbreviations: HE, hematoxylin and eosin; NLCs, nanolipoidal carriers; CAP, capsaicin.

skin and the $1.5 \mathrm{mg} / \mathrm{mL}$ capsaicin solution showed slight irritation on normal skin and moderate irritation on injured skin. The $1.5 \mathrm{mg} / \mathrm{mL}$ capsaicin-loaded NLCs and $1.5 \mathrm{mg} / \mathrm{mL}$ capsaicin-loaded NLCs gel showed slightly less irritation than the capsaicin solution groups. The irritation increased when the concentration of capsaicin was increased, and the skin irritation could recover overnight except for the $1.5 \mathrm{mg} / \mathrm{mL}$ capsaicin solution on injured skin. The results demonstrated that the irritation induced by all the capsaicin preparations after 3 days of continuous administration was reversible, and application of the NLCs could reduce skin irritation induced by capsaicin, especially for high concentration (Tables S3 and S4).

According to the histological observation by hematoxylin and eosin (HE) staining, it was found that both the normal skin and injured skin treated with different capsaicin-based preparations show no inflammation. Inflammatory cells could not be seen in the HE staining. All the groups showed similar skin morphology when compared with the normal skin, which meant that the slight skin irritation induced by the different capsaicin-based preparations was all reversible after 3 days of observation (Figure 7). The difference between the results from the evaluation score and HE staining was mainly due to the 3 days of observation. After 3 days of repair, the initial acute inflammation caused by capsaicin was not obvious and could not be distinguished in the HE staining. Thus, it can be concluded through these collective findings that although all the irritation could recover after several days of repair, the injured skin sites should be avoided when capsaicin-based preparations are applied to the skin. Using NLCs as the carrier for topical delivery of capsaicin could significantly reduce the initial skin irritation caused by capsaicin to improve the compliance of patients in clinical use.

\section{Conclusion}

For the application of capsaicin, NLCs were developed for delivering capsaicin in the deep dermal region in order to reduce irritation. The carriers were modified by a hot melt homogenization technique and optimized using the BoxBehnken design. The studies demonstrated that optimal NLCs can significantly enhance the cumulative drug permeated, permeation flux, and retention of capsaicin due to the nano-size, composition, and sustained release of NLCs and mainly through the appendages route. Furthermore, capsaicin-loaded NLCs were incorporated into carbopol, enhancing the skin contact of the formulation and the ease of application without altering the release or skin distribution and permeation characteristics of the NLCs. Capsaicinloaded NLCs and capsaicin-loaded NLCs gel could achieve the analgesic and anti-inflammatory effect with the reduction 
of skin irritation. The present results demonstrate that capsaicin-loaded NLCs could be a promising modality for topical capsaicin administration.

\section{Disclosure}

The authors report no conflicts of interest in this work.

\section{References}

1. Andersen HH, Sand C, Elberling J. [Effective pain relief of postherpetic neuralgia with capsaicin patch]. Ugeskr Laeger. 2016;178(5): V10150846. Danish.

2. Kumar Sarwa K, Rudrapal M, Mazumder B. Topical ethosomal capsaicin attenuates edema and nociception in arthritic rats. Drug Deliv. 2015;22(8):1043-1052.

3. Rains C, Bryson HM. Topical capsaicin. A review of its pharmacological properties and therapeutic potential in post-herpetic neuralgia, diabetic neuropathy and osteoarthritis. Drugs Aging. 1995;7(4):317-328.

4. Hayman M, Kam PCA. Capsaicin: a review of its pharmacology and clinical applications. Curr Anesth Crit Care. 2008;19(5-6):338-343.

5. Chhabra N, Aseri ML, Goyal V, Sankhla S. Capsaicin: a promising therapy - a critical reappraisal. Int J Nutr Pharmacol Neurol Dis. 2012;2(1): $8-15$.

6. Mankowski C, Patel S, Trueman D, Bentley A, Poole C. Cost-effectiveness of capsaicin $8 \%$ patch compared with pregabalin for the treatment of patients with peripheral neuropathic pain in Scotland. PLoS One. 2016; 11(3): 0150973.

7. Xing F, Cheng G, Yi K, Ma L. Nanoencapsulation of capsaicin by complex coacervation of gelatin, acacia, and tannins. J Appl Polym Sci. 2005;96(6):2225-2229.

8. Sarwa KK, Mazumder B, Rudrapal M, Verma VK. Potential of capsaicin-loaded transfersomes in arthritic rats. Drug Deliv. 2015; 22(5):638-646.

9. Chaiyasit K, Khovidhunkit W, Wittayalertpanya S. Pharmacokinetic and the effect of capsaicin in Capsicum frutescens on decreasing plasma glucose level. J Med Assoc Thai. 2009;92(1):108-113.

10. Chanda S, Bashir M, Babbar S, Koganti A, Bley K. In vitro hepatic and skin metabolism of capsaicin. Drug Metab Dispos. 2008;36(4): 670-675.

11. Wang Y, Hong C, Chiu W, Fang J. In vitro and in vivo evaluations of topically applied capsaicin and nonivamide from hydrogels. Int $J$ Pharm. 2001;224(1-2):89-104.

12. Mason L, Moore RA, Edwards JE, Mcquay HJ, Derry S, Wiffen PJ. Systematic review of topical capsaicin for the treatment of chronic pain. BMJ. 2004;328(7446):991-994.

13. Bode AM, Dong Z. The two faces of capsaicin. Cancer Res. 2011; 71(8):2809-2814.

14. Derry S, Moore RA. Topical capsaicin (high concentration) for chronic neuropathic pain in adults. Cochrane Database Syst Rev. 2013;2(2):24.

15. Lowes MA, Bowcock AM, Krueger JG. Pathogenesis and therapy of psoriasis. Nature. 2007;445(7130):866-873.

16. Raza K, Shareef MA, Singal P, Sharma G, Negi P, Katare OP. Lipidbased capsaicin-loaded nano-colloidal biocompatible topical carriers with enhanced analgesic potential and decreased dermal irritation. J Liposome Res. 2014;24(4):290-296.

17. Iqbal MA, Md S, Sahni JK, Baboota S, Dang S, Ali J. Nanostructured lipid carriers system: recent advances in drug delivery. J Drug Target. 2012;20(10):813-830.

18. Zauner W, Farrow NA, Haines AMR. In vitro uptake of polystyrene microspheres: effect of particle size, cell line and cell density. J Control Release. 2001;71(1):39-51.

19. Yoon G, Jin WP, Yoon IS. Solid lipid nanoparticles (SLNs) and nanostructured lipid carriers (NLCs): recent advances in drug delivery. J Pharm Investig. 2013;43(5):353-362.
20. Müller RH, Radtke M, Wissing SA. Nanostructured lipid matrices for improved microencapsulation of drugs. Int J Pharm. 2002;242(1-2): 121-128.

21. Sivaramakrishnan R, Nakamura C, Mehnert W, Korting HC, Kramer KD, Schäfer-Korting M. Glucocorticoid entrapment into lipid carriers - characterisation by parelectric spectroscopy and influence on dermal uptake. J Control Release. 2004;97(3):493.

22. Gupta M, Agrawal U, Vyas SP. Nanocarrier-based topical drug delivery for the treatment of skin diseases. Expert Opin Drug Deliv. 2012;9(7):783-804.

23. Touska F, Marsakova L, Teisinger J, Vlachova V. A “cute" desensitization of TRPV1. Curr Pharm Biotechnol. 2011;12(1):122-129.

24. Muller RH, Shegokar R, Keck CM. 20 years of lipid nanoparticles (SLN and NLC): present state of development and industrial applications. Curr Drug Discov Technol. 2011;8(3):207-227.

25. Shah PP, Desai PR, Channer D, Singh M. Enhanced skin permeation using polyarginine modified nanostructured lipid carriers. J Control Release. 2012;161(3):735-745.

26. Ahad A, Aqil M, Kohli K, Sultana Y, Mujeeb M, Ali A. Formulation and optimization of nanotransfersomes using experimental design technique for accentuated transdermal delivery of valsartan. Nanomedicine. 2011;8(2):237-249.

27. Ahad A, Aqil M, Kohli K, Sultana Y, Mujeeb M. Design, formulation and optimization of valsartan transdermal gel containing iso-eucalyptol as novel permeation enhancer: preclinical assessment of pharmacokinetics in Wistar albino rats. Expert Opin Drug Deliv. 2014;11(8): $1149-1162$.

28. Pradhan M, Singh D, Murthy SN, Singh MR. Design, characterization and skin permeating potential of Fluocinolone acetonide loaded nanostructured lipid carriers for topical treatment of psoriasis. Steroids. 2015;101:56-63.

29. Gokce EH, Korkmaz E, Dellera E, Sandri G, Bonferoni MC, Ozer O. Resveratrol-loaded solid lipid nanoparticles versus nanostructured lipid carriers: evaluation of antioxidant potential for dermal applications. Int J Nanomedicine. 2012;7:1841-1850.

30. Chen ZL, Huang M, Wang XR, et al. Transferrin-modified liposome promotes alpha-mangostin to penetrate the blood-brain barrier. Nanomedicine. 2016;12(2):421-430.

31. zur Muhlen A, Schwarz C, Mehnert W. Solid lipid nanoparticles (SLN) for controlled drug delivery - drug release and release mechanism. Eur J Pharm Biopharm. 1998;45(2):149-155.

32. Nastassja L, Vicki C, Rebekah D. Cytotoxicity of nanoparticles. Small. 2008;4(1):26-49.

33. Desai PR, Marepally S, Patel AR, Voshavar C, Chaudhuri A, Singh M. Topical delivery of anti-TNF $\alpha$ siRNA and capsaicin via novel lipidpolymer hybrid nanoparticles efficiently inhibits skin inflammation in vivo. J Control Release. 2013;170(1):51-63.

34. Shah PP, Desai PR, Singh M. Effect of oleic acid modified polymeric bilayered nanoparticles on percutaneous delivery of spantide II and ketoprofen. J Control Release. 2012;158(2):336-345.

35. Ming C, Gupta V, Anselmo AC, Muraski JA, Mitragotri S. Topical delivery of hyaluronic acid into skin using SPACE-peptide carriers. J Control Release. 2013;173(1):67-74.

36. Li N, Peng LH, Chen X, Nakagawa S, Gao JQ. Effective transcutaneous immunization by antigen-loaded flexible liposome in vivo. Int $J$ Nanomedicine. 2010;6(6):3241-3250.

37. Fell GL, Robinson KC, Mao J, Woolf CJ, Fisher DE. Skin beta-endorphin mediates addiction to UV light. Cell. 2014;157(7):1527-1534.

38. Peng LH, Wei W, Shan YH, Chong YS, Yu L, Gao JQ. Sustained release of piroxicam from solid lipid nanoparticle as an effective anti-inflammatory therapeutics in vivo. Drug Dev Ind Pharm. 2016; 43(1): $1-25$

39. Morris CJ. Carrageenan-induced paw edema in the rat and mouse. Methods Mol Biol. 2003;225:115-121.

40. Draize J, Woodard G, Calvery H. Methods for the study of irritation and toxicity of substances applied topically to the skin and mucous membranes. Fertil Steril. 2015;6(3):105-107. 
41. Mitri K, Shegokar R, Gohla S, Anselmi C, Müller RH. Lipid nanocarriers for dermal delivery of lutein: preparation, characterization, stability and performance. Int J Pharm. 2011;414(1-2):267-275.

42. Ferreira M, Chaves LL, Lima SA, Reis S. Optimization of nanostructured lipid carriers loaded with methotrexate: a tool for inflammatory and cancer therapy. Int J Pharm. 2015;492(1-2):65-72.

43. Garg NK, Tyagi RK, Singh B, et al. Nanostructured lipid carrier mediates effective delivery of methotrexate to induce apoptosis of rheumatoid arthritis via NF-kb and FOXO1. Int J Pharm. 2016;499(1-2): 301-320.

44. Jain A, Kesharwani P, Garg NK, et al. Galactose engineered solid lipid nanoparticles for targeted delivery of doxorubicin. Colloids Surf B Biointerfaces. 2015;134:47-58.

45. Chen X, Peng LH, Shan YH, et al. Astragaloside IV-loaded nanoparticleenriched hydrogel induces wound healing and anti-scar activity through topical delivery. Int J Pharm. 2013;447(1-2):171-181.
46. Kohli AK, Alpar HO. Potential use of nanoparticles for transcutaneous vaccine delivery: effect of particle size and charge. Int J Pharm. 2004;275(1-2):13-17.

47. Trommer H, Neubert RH. Overcoming the stratum corneum: the modulation of skin penetration. A review. Skin Pharmacol Physiol. 2006;19(2):106-121.

48. Jenning V, Gysler A, Schäfer-Korting M, Gohla SH. Vitamin A loaded solid lipid nanoparticles for topical use: occlusive properties and drug targeting to the upper skin. Eur J Pharm Biopharm. 2000; 49(3):211-218.

49. Huang J, Fan Y, Jia Y, Hong Y. Antagonism of 5-HT(2A) receptors inhibits the expression of pronociceptive mediator and enhances endogenous opioid mechanism in carrageenan-induced inflammation in rats. Eur J Pharmacol. 2011;654(1):33-41. 


\section{Supplementary materials}

Table SI Variables and their levels in the Box-Behnken design

\begin{tabular}{llll}
\hline Variables & Levels & $\mathbf{0}$ \\
\cline { 2 - 3 } & $-\mathbf{I}$ & \\
\hline Independent variables & & 0.5 & $+\mathbf{I}$ \\
$\mathrm{X}_{1}$ (solid lipid:amount of lipid $\left.[\mathrm{w} / \mathrm{w}]\right)$ & 0.1 & 0.9 \\
$\mathrm{X}_{2}$ (total amount of lipid $\left.[\mathrm{w} / \mathrm{v}]\right)$ & 0.8 & 0.3 \\
$\mathrm{X}_{3}$ (total amount of surfactant $\left.[\mathrm{w} / \mathrm{v}]\right)$ & 0.2 & \\
\hline Dependent variables & Constraints & \\
\hline$Y_{1}$ (entrapment efficiency) & Maximize & \\
$Y_{2}$ (particle size) & Minimize & \\
\hline
\end{tabular}

Table S2 Box-Behnken design for the study of three experimental factors in coded and actual levels with experimental results

\begin{tabular}{|c|c|c|c|c|c|}
\hline \multirow{2}{*}{$\begin{array}{l}\text { Formulation } \\
\text { code }\end{array}$} & \multicolumn{3}{|c|}{ Actual value of variables } & \multicolumn{2}{|c|}{ Response values } \\
\hline & $\mathbf{x}_{1}$ & $\mathbf{x}_{2}$ & $x_{3}$ & EE (\%) & PS (nm) \\
\hline $\mathrm{FI}$ & 0.1 & 0.8 & 0.3 & 84.5 & 150 \\
\hline F2 & 0.9 & 0.8 & 0.3 & 85.6 & 123 \\
\hline F3 & 0.1 & 1 & 0.3 & 85.1 & 153 \\
\hline F4 & 0.9 & I & 0.3 & 89.8 & 180 \\
\hline F5 & 0.1 & 0.9 & 0.2 & 81.9 & 169 \\
\hline F6 & 0.9 & 0.9 & 0.2 & 85.1 & 199 \\
\hline F7 & 0.1 & 0.9 & 0.4 & 88.1 & 142 \\
\hline F8 & 0.9 & 0.9 & 0.4 & 86.9 & 128 \\
\hline F9 & 0.5 & 0.8 & 0.2 & 84.2 & 130 \\
\hline FIO & 0.5 & 1 & 0.2 & 82.8 & 164 \\
\hline FII & 0.5 & 0.8 & 0.4 & 88.6 & 120 \\
\hline $\mathrm{FI} 2$ & 0.5 & 1 & 0.4 & 89.4 & 146 \\
\hline $\mathrm{FI3}$ & 0.5 & 0.9 & 0.3 & 89.5 & 123 \\
\hline $\mathrm{FI} 4$ & 0.5 & 0.9 & 0.3 & 91.6 & 120 \\
\hline $\mathrm{FI5}$ & 0.5 & 0.9 & 0.3 & 90.4 & 125 \\
\hline
\end{tabular}

Abbreviations: F, formulation; EE, entrapment efficiency; PS, particle size.

Table S3 Score for skin irritation study on normal rabbit skin calculated after 3 days of administration and 3 days of observation

\begin{tabular}{lllll}
\hline Category for reaction & $\begin{array}{l}\mathbf{l} .5 \mathbf{~ m g} / \mathbf{m L} \\
\text { CAP solution }\end{array}$ & $\begin{array}{l}\mathbf{0 . 7 5} \mathbf{~ m g} / \mathbf{m L} \\
\text { CAP solution }\end{array}$ & $\begin{array}{l}\text { I.5 } \mathbf{~ m g} / \mathbf{m L} \text { CAP- } \\
\text { loaded NLCs }\end{array}$ & $\begin{array}{l}\text { I.5 } \mathbf{~ m g / m L ~ C A P - ~} \\
\text { loaded NLCs gel }\end{array}$ \\
\hline I h after first administration & 2 & 2 & $\mathrm{I}$ & 0 \\
Before second administration & $\mathrm{I}$ & $\mathrm{I}$ & 0 & 0 \\
I h after second administration & 2 & $\mathrm{I}$ & $\mathrm{I}$ & $\mathrm{I}$ \\
Before third administration & $\mathrm{I}$ & $\mathrm{I}$ & 0 & 0 \\
I h after third administration & 2 & 2 & $\mathrm{I}$ & $\mathrm{I}$ \\
24 h after third administration & 2 & $\mathrm{I}$ & 0 & 0 \\
$48 \mathrm{~h}$ after third administration & 0 & 0 & 0 & 0 \\
$72 \mathrm{~h}$ after third administration & 0 & 0 & 0 & 0 \\
\hline
\end{tabular}

Note: 0-0.49: non-irritation, 0.5-2.99: slight irritation, 3.0-5.99: moderate irritation, 6.0-8.0: strong irritation.

Abbreviations: CAP, capsaicin; NLCs, nanolipoidal carriers. 
Table S4 Score for skin irritation study on injured rabbit skin calculated after 3 days of administration and 3 days of observation

\begin{tabular}{|c|c|c|c|c|}
\hline Category for reaction & $\begin{array}{l}1.5 \mathrm{mg} / \mathrm{mL} \\
\text { CAP solution }\end{array}$ & $\begin{array}{l}0.75 \mathrm{mg} / \mathrm{mL} \\
\text { CAP solution }\end{array}$ & $\begin{array}{l}1.5 \mathrm{mg} / \mathrm{mL} \text { CAP- } \\
\text { loaded NLCs }\end{array}$ & $\begin{array}{l}1.5 \mathrm{mg} / \mathrm{mL} \text { CAP- } \\
\text { loaded NLCs gel }\end{array}$ \\
\hline I h after first administration & 2 & 2 & I & 0 \\
\hline Before second administration & I & I & 0 & 0 \\
\hline $\mathrm{I} \mathrm{h}$ after second administration & 2 & I & I & I \\
\hline Before third administration & I & I & 0 & 0 \\
\hline I $\mathrm{h}$ after third administration & 2 & 2 & 2 & 1 \\
\hline $24 \mathrm{~h}$ after third administration & 4 & I & 0 & 0 \\
\hline $48 \mathrm{~h}$ after third administration & 4 & 0 & 0 & 0 \\
\hline $72 \mathrm{~h}$ after third administration & 4 & 0 & 0 & 0 \\
\hline
\end{tabular}

Note: 0-0.49: non-irritation, 0.5-2.99: slight irritation, 3.0-5.99: moderate irritation, 6.0-8.0: strong irritation.

Abbreviations: CAP, capsaicin; NLCs, nanolipoidal carriers.

\section{Publish your work in this journal}

The International Journal of Nanomedicine is an international, peerreviewed journal focusing on the application of nanotechnology in diagnostics, therapeutics, and drug delivery systems throughout the biomedical field. This journal is indexed on PubMed Central, MedLine, CAS, SciSearch ${ }^{\circledR}$, Current Contents ${ }^{\circledR} /$ Clinical Medicine,
Journal Citation Reports/Science Edition, EMBase, Scopus and the Elsevier Bibliographic databases. The manuscript management system is completely online and includes a very quick and fair peer-review system, which is all easy to use. Visit http://www.dovepress.com/ testimonials.php to read real quotes from published authors.

Submit your manuscript here: http://www.dovepress.com/international-journal-of-nanomedicine-journal 